\title{
Abstracts of the European Society of Clinical Pharmacy International Workshop on Malabsorption and Malnutrition, A Challenge for Clinical Pharmacists, 26-27 April 2021
}

(C) Springer Nature Switzerland AG 2021

\section{Oral Communications}

\section{OR01.1}

Analyzing and optimizing the current procedure for preparing and administering drugs to patients with an enteric tube and/ or dysphagia

L. Mattar ${ }^{1, *}$, M. Bourton ${ }^{1}$, M. Mattens ${ }^{1}$

${ }^{1}$ Pharmacy, Sankt Nikolaus Hospital, Eupen, Belgium

Background and Objective: Administering drugs to patients with an enteric tube and/or dysphagia is a current problem in hospitals. Nursing staff change the pharmaceutical formulation of drugs without knowing the possible consequences. A procedure for the administration of medicines through an enteric tube or for patients with dysphagia was introduced in our institution in 2012; a 180-bed secondary care general hospital in Belgium; but its correct use has not been evaluated so far.

The aim of the study was to evaluate the correct use of this procedure by nursing staff before and after implementing improvements. A secondary objective was to introduce a back office clinical pharmacy service for these patients.

Design: A prospective and observational study was performed on drug preparation and administration through an enteric tube or for patients with dysphagia between July-August 2018 and, after implementing ways of improvements, between February-March 2019. In addition, a survey on the knowledge and correct use of the procedure among a staff of 132 nurses was carried out for both periods of time.

Also, screenings for drug related problems were made and pharmaceutical interventions recorded in the patient's electronic record. Their acceptance rate was verified afterwards.

Results: None of the survey participants $(\mathrm{N}=64)$ followed the procedure correctly and even $22(35,5 \%)$ were not aware of its existence. After introducing and promoting improvements, one of three nurses $(\mathrm{N}=10)$ checked the information on the patient record before preparing and administering each drug. Nurse satisfaction also increased by 34,5 percent points. However, not every step of the procedure could be improved. A total of 31 pharmaceutical interventions were made, whereof $51,6 \%$ were accepted by the physicians. Conclusion: By optimizing the procedure, some steps of drug preparation and administration may be improved. Regular audits and improvements should be performed in order to guarantee quality of treatment. This is the clinical pharmacist's responsibility.

Disclosure of Interest: None Declared.

\section{OR01.2}

Analysis of the adequacy of the prescription of thickeners in a health area and its economic impact

B. Nucete $^{1, *}$, F. Malpica ${ }^{1}$, M. Camacho' ${ }^{1}$

${ }^{1}$ Pharmacy, Hospital of Motril. Granada Southern Health Management Area, Motril, Spain

Background and Objective: According to national legislation, thickening agents (TA) should be prescribed for neurological or excepcionally motor dysphagia, with the possibility of eating solid foods without risk of aspiration, but suffers risk for liquid foods, when they cannot be thickened with ordinary alternatives, in order to prevent or delay the use of a tube or gastrostomy.

To analyze the characteristics of patients prescribed TM, the indications and the economic impact of these prescriptions.

Design: Observational, retrospective study which included all patients who have been given a TA at the pharmacy, from October to December 2020 in the health area of our hospital. Patients were selected thanks to a local application (MicroStrategy $\left.{ }^{\circledR}\right)$. Data were collected through the electronic medical report $(\mathrm{MH})\left(\right.$ Diraya $\left.{ }^{\circledR}\right)$, and reviewing electronic prescriptions (Recipe XXI $\left.{ }^{\circledR}\right)$. A database was developed analyzing: age, physicians/patients provenance (primary (PC) or specialized care (SC), diagnosis, neurological/motor cause, nutritional evaluation (NE) and economic expense.

Results: During the period of study, 133 patients were included, $(57,14 \%$ female). Mean age: $77.16 \pm 16.48$ years. Need for a TM detected in PC: $75.79 \%$ of patients but prescribed by SC physicians: $99.25 \%$. Diagnoses according to criteria: degenerative process of the central nervous system (DPCNS) $(\mathrm{n}=69,51.88 \%)$, stroke $(\mathrm{n}=32$, $24.06 \%)$, head/neck tumor $(\mathrm{n}=4,3.01 \%)$ (motor cause), mental retardation $(n=7,5.26 \%)$, cerebral palsy $(n=5,3.76 \%)$, sequelae of traumatic diseases $(\mathrm{n}=2,1.50 \%)$, non-tumor esophageal stricture $(\mathrm{n}=1,0.75 \%)$. Prescription not comply with the legislation: $5.26 \%$. Diagnosis not recorded in the $\mathrm{MH}: 4.51 \%$. Fluid dysphagia (FD) recorded in the HC: $34.47 \%$. $63.10 \%$ did not specify the type. $2.43 \%$ referred risk of aspiration to solids. NE was performed in $8.27 \%$ of the patients. Average spending per patient in a quarter: 
$127.53 € \pm 66.19$. Total expenditure on thickeners estimated per year: $67.846,68 €$.

Conclusion: Most of FD are found in PC, but treated by a SC physician. The main cause of FD was DPCNS. It is important to evaluate and reflect in the $\mathrm{CH}$ the tolerance without risk of the patient to solids. Since most are patients at risk of malnutrition, NE should be greatly increased. In general, TA prescriptions meet the criteria, but due to their economic and social impact, it should be given the importance that corresponds to them.

Disclosure of Interest: None Declared.

\section{OR01.3}

Nutrition in intensive care: impact of the clinical pharmacist on the optimization of daily prescriptions

\section{A. Cappe ${ }^{1, *}$, H. Nguyen ${ }^{2}$, C. Omnes ${ }^{1}$, F. Tinturier ${ }^{3}$, A. Mary ${ }^{1}$}

${ }^{1}$ Clinical Pharmacy Department, ${ }^{2}$ Pharmacy, ${ }^{3}$ Surgical intensive care, CHU Amiens Picardie, Amiens, France

Background and Objective: Non-optimal nutritional management can worsen the prognosis of the patient in intensive care. The patient's nutritional status and nutritional prescriptions are reassessed daily, with separate caloric and vitamin requirements depending on the patient's condition. The pharmacist may suggest dosage adjustments or therapeutic changes based on the patient's condition. These pharmaceutical interventions (PIs) may concern artificial nutrition (enteral, parenteral) or co-prescribed vitamins.

The objective is to collect retrospectively over 3 years the PIs in surgical intensive care benefiting from pharmaceutical analysis within the department.

Design: Extraction of PIs over 3 years on the Act-IP ${ }^{\circledR}$ software, ratio to the number of patients who stayed more than 3 days in surgical intensive care unit and received at least one nutritional prescription. The CLEO ${ }^{\circledR}$ scale was used by clinical pharmacists to assess clinical, economic and organisational impact of PIs.

Results: In three years, 773 PIs have been transmitted on nutrition and co-prescribed vitamins in surgical intensive care unit:

58 Pis concerned nutrients ( 9 and 49 for enteral and parenteral nutrition respectively). $31 \%$ of the PIs on nutrition concerned the adjustment of calorie and protein dosage according to the patient's requirements (septic shock, malnutrition, extra-renal epuration), 25\% concerned a possible addition or relay of nutrition.

715 of the PIs concerned vitamins. $77 \%$ of these were an omission of prescription in the case of parenteral nutrition, or a vitamin requirement not covered for a particular situation (cirrhotic patient, patient in septic shock, extra-renal epuration). The remaining PIs targeted the discontinuation of unnecessary vitamins in case of enteral nutrition relays (which already contain the vitamins) or the excessive duration of vitamin therapy.

Over the study period, 1803 patients were hospitalised in surgical intensive care with artificial nutrition, for a stay of more than 3 days. The 773 PIs concerned 383 patients, i.e. $21 \%$ of the patients during this period, and a mean PIs of 0.42 .

IPs acceptance rate has been excellent (96.8\%). Pharmacists rated the impact of these PIs as moderate and major in $48.6 \%$ and $29.5 \%$ of cases, respectively. The economic impact was negative in $39.3 \%$ of cases, zero in $26.9 \%$, and in favour of cost reduction in $30.9 \%$ of cases. PIs had a favourable organizational impact in $20 \%$ of cases; unfavourable in $7.2 \%$, and neutral in $71.4 \%$ of PIs.

Conclusion: The medical prescription of the diet is fundamental, and ensures the patient's daily calorie requirements are met. However, a large part of the interventions carried out do not concern nutrition but the co-prescribed vitamins. The clinical pharmacist can contribute to this both through daily analysis and by constantly monitoring the latest scientific data on the subject, with a high level of acceptance of the PIs.

Disclosure of Interest: None Declared.

\section{OR02.1}

Health and nutritional status of Croatian older people: preliminary results from the Croatian sample of the EUROAGEISM ESR7 H2020 project

I. Kummer ${ }^{1,2, *}$, M. Držaić ${ }^{1}$, I. Bužančić ${ }^{1}$, M. Marušić ${ }^{3}$, D. Butković $^{4}$, M. Kolarić ${ }^{5}$, H. Jurinić ${ }^{6}$, M. Matković ${ }^{7}$, L. Brečević ${ }^{8}$, T. Buršić ${ }^{8}$, M. Ortner Hadžiabdić ${ }^{8}$, J. Brkić ${ }^{2}$, D. Fialová, ${ }^{2,9}$

${ }^{1}$ Zagreb City Pharmacies, Zagreb, Croatia, ${ }^{2}$ Department of Social and Clinical Pharmacy, Faculty of Pharmacy in Hradec Králové, Charles University, Hradec Králové, Czech Republic, ${ }^{3}$ Community Pharmacy Sonja Grbac Stublić, Zagreb, ${ }^{4}$ Community Pharmacy chain Lijek Đakovo, Đakovo, ${ }^{5}$ Community Pharmacy Poljak-Radnić, Sibinj, ${ }^{6}$ Community Pharmacy Šibalić, Vinkovci, ${ }^{7}$ Community Pharmacy chain Ljekarna Pablo, Rijeka, ${ }^{8}$ Faculty of Pharmacy and Biochemistry, University of Zagreb, Zagreb, Croatia, ${ }^{9}$ Department of Geriatrics and Gerontology, 1st Faculty of Medicine, Prague, Czech Republic

Background and Objective: The aim of the study was to determine the health and nutritional status of the Croatian older population visiting community pharmacies, using the methods of comprehensive geriatric assessment.

Setting and Method: The research was conducted as part of the EUROAGEISM H2020 ESR 7 project and was designed as a crosssectional study. Data were collected in community pharmacies in the City of Zagreb, and regions of Slavonia and Istria. Subjects who met the inclusion criteria (age $\geq 65$ years and at least one drug in therapy) and voluntarily signed informed consent were interviewed using a structured, standardized questionnaire developed for the EUROAGEISM H2020 ESR 7 international project.

Main outcome measures: Nutritional status, assessment of frailty syndrome, cognitive status, mood, activities of daily living test (ADL) and mobility and strength.

Results: The study included 208 participants (64.8\% women, median age 73 years (interquartile range (IQR) 69-80)) of which $20.7 \%$ being older than 80 years. Median body mass index was 27.6 (IQR 24.8-30.9) with $73.8 \%$ being overweight or obese and $3.9 \%$ being underweight. $31.9 \%$ reported food intake decline in the past three months, while $27.6 \%$ participant reported weight loss during the same period. In performing daily activities, most respondents showed independence $(80.5 \%-96.7 \%$, depending on the assessed activity). $23.8 \%$ of participants were vulnerable or mildly-moderately frail, $2 \%$ were seriously frail, while others were fit or managing well. The majority were of good cognitive status (93\%), but $22.9 \%$ of them reported problems with short-term memory. Depressed mood was observed in $43.8 \%$ of respondents.

Conclusion: Among the older community-dwelling population in Croatia, we observed that most people were mobile and could perform daily activities independently, did not have a pronounced frailty syndrome or significantly impaired cognitive status. Depressed mood has been identified as the most common problem, followed by food intake decline.

Grant support: the EUROAGEISM H2020-MCSF-ITN-764632 project, INOMED project (CZ.02.1.01/0.0/0.0/18_069/0010046), PROGRESS Q42, Faculty of Pharmacy, Charles University (KSKF-2 Assoc. Prof. Fialova) and SVV 260417.

Disclosure of Interest: None Declared. 


\section{OR02.2}

Pharmacodynamics of drugs in patients with short bowel syndrome: dose adjustment and guidelines

\section{J. Dupuy ${ }^{1, *}$}

${ }^{1}$ Pharmacie, Centre Hospitalier Intercommunal des Vallées de l'Ariège, Saint-Jean-de-Verges, France

Background and Objective: Short bowel syndrome is a rare disease due to a 150 to $200 \mathrm{~cm}$ intestinal resection of post duodenal gut. Drugs are directly impacted by digestive malabsorption resulting from digestive ph and solubility modifications. Gut metabolization is also enhanced and adaptation of the medicinal dosage is required for affected patients.Objective is to set drug guidelines, dosage and galenic advices to prescribers for patients with short bowel syndrome. Design: I conducted an exhaustive literature review of available publications on large jejunum resections. Then I developed a cross tabulation (classified by drugs international nonproprietary name, princeps, therapeutic class and recommended use) in order to help gastroenterology physicians for dosage adjustment. Finally, i gathered medicine galenic data to elaborate guidelines.

Results: Guidelines batch 49 medications often prescribed in short bowel syndrome. Drugs for digestive system (proton-pump inhibitors, gut motility drugs, antidiarrheal microorganisms) were the most commonly used (26,5\%), along with antihypertensive drugs (16,3\%), vitamins and minerals (14,3\%), antithrombotics/antihemorrhagics $(12,2 \%)$ and thyroid hormones $(8,2 \%)$. Talking about galenic, it is wise to use solubilized forms (oral solutions, syrups) and orodispersible tablets. Capsules, matrix tablets, extended release and delayed release forms must be avoided. Molecules already dissolved will be faster absorbed but liquid forms are not advised for patients with significant diarrhea ( $>8$ stools/day): gut motility is enhanced and the intestinal residence time falls. Sorbitol, as excipient, in not recommended for may cause osmotic diarrhea. Transdermal, sublingual, nasal and rectal routes (without diarrhea) are privileged but parenteral administration is always the last resort.

Conclusion: This cross tabulation summary allows ease physicians with latest short bowel syndrome dose adjustment and recommendations. This work is an example of multidisciplinary collaboration necessary to optimize patient's pharmaceutical and therapeutic cares. I plan to study on others frequently prescribed drugs in short bowel syndrome in order to provide physicians with new reliable data for dosage adjustment.

Disclosure of Interest: None Declared.

\section{OR02.3}

Evaluating the role and integration of general practice pharmacists in England: a mixed-method cross-sectional study

A. Alshehri ${ }^{1,2, *}$, E. Cheema ${ }^{1}$, A. Yahyouche ${ }^{1}$, M. S. Haque ${ }^{3}$, Z. Jalal $^{1}$

${ }^{1}$ School of Pharmacy, University of Birmingham, Birmingham, United Kingdom, ${ }^{2}$ Pharmacy School, Department of Clinical, Taif University, Taif, Saudi Arabia, ${ }^{3}$ Institute of Applied Health research, University of Birmingham, Birmingham, United Kingdom

Background and Objective: Since the year 2015, the National Health Service England has facilitated the recruitment of pharmacists in general practice to reduce the workload of general practitioners. The role of pharmacists is therefore, expected to become more clinical and patient oriented. However, little is known about the current roles performed and the level of integration of pharmacists in general practice. This study aims to assess the role and types of activities undertaken by practice pharmacists and thier integration into practice across England.

Setting and Method: A mixed-method cross-sectional survey of general practice pharmacists in England. The study used both online and paper-based questionnaires for a period of six months between May and November 2019. Survey items included demographics, roles performed in general practice and integration into practice including practice environment and available support. Quantitative data were analysed using descriptive statistics and $95 \%$ confidence intervals. Qualitative data were analysed thematically, to identify the perceptions of pharmacists about their current roles and the main barriers and facilitators of integration into general practice.

Main outcome measures: The types of activities undertaken by practice pharmacists and their level of integration into practice. Integration was assessed via practice environment (number of practice meetings attended, formal appraisal conducted and physical workspace) and pharmacists' satisfaction about their integration into practice and support received from GP practice team.

Results: 195 participants completed the questionnaire. Of these, three quarters of pharmacists (76\%) had only been in general practice since 2015. Most pharmacists (81\%) were independent prescribers (IPs). The most reported pharmacists' roles were medicine reconciliation (95\%), telephone support for patients $(95 \%)$ and face-to-face medication review (91\%). $82 \%$ (95\% CI: $76-86.8 \%$ ) were satisfied with their overall integration into the general practice. The lowest level of satisfaction was found to be associated with working closely with the other members of the multidisciplinary team $(76.5 \%, 95 \% \mathrm{CI}$ : $67-81.8 \%)$ and with the support received from GPs $(77.5 \%, 95 \% \mathrm{CI}$ : $71-82.7 \%)$. Half of pharmacists $(45 \%)$ were working in a shared office or at a hot desk and $9 \%$ had no designated workspace. Independent prescribers had access to a convenient workplace (diff $=23 \%, 95 \%$ CI: 5-37\%).

Conclusion: General practice pharmacists are expected to deliver a range of clinical and patient-oriented roles in England. The findings highlight the issues of integration into general practice and its environment. Integration could be particularly challenging for those working part-time, covering multiple practices, who are not IPs and those who have less experience in general practice. These findings have important considerations when developing future roles of pharmacists in general practice.

Disclosure of Interest: None Declared.

\section{Posters}

\section{PP01}

Ruxolitinib exposure in patients with acute and chronic graft versus host disease-a prospective single-center trial

F. Aghai ${ }^{1, *}$, N. Isberner ${ }^{1}$, S. Zimmermann ${ }^{2}$, M. Kurlbaum ${ }^{3}$, S. Kraus $^{4}$, G. U. Grigoleit ${ }^{5}$, O. Scherf-Clavel ${ }^{2}$, H. Klinker ${ }^{1}$

${ }^{1}$ Internal Medicine II, Medical Center, University of Wuerzburg,

${ }^{2}$ Institute of Pharmacy and Food Chemistry, University of Wuerzburg, Wuerzburg, ${ }^{3}$ Core Unit Clinical Mass Spectrometry, Medical Center, University of Wuerzburg, Wurzburg, ${ }^{4}$ Internal Medicine II, Medical Center, University of Wuerzburg, Wuerzburg, ${ }^{5}$ Medicine II, Helios Hospital Duisburg, Duisburg, Germany

Background and Objective: Graft versus host disease (GvHD) remains one of the major causes for morbidity and mortality after allogeneic hematopoietic stem cell transplantation (allo-HSCT). Ruxolitinib is a Janus Kinase $1 / 2$ inhibitor and was approved by the FDA for acute GvHD. Ruxolitinib is predominantly metabolized by CYP3A4, to a lesser extent by CYP2C9 and has a pH-dependent solubility. Comedication, genetic polymorphisms and other patient 
characteristics may influence Ruxolitinib metabolism. Hepatic or renal impairment and gastrointestinal GvHD after allo-HSCT may also alter Ruxolitinib exposure. As knowledge of Ruxolitinib exposure in patients with chronic or acute GvHD is scarce, we report analysis of plasma levels in daily clinical practice and evaluate the use of therapeutic drug monitoring (TDM).

Setting and Method: Ruxolitinib plasma concentrations were determined using a fully validated liquid chromatography mass spectrometry method. An exploratory population pharmacokinetic analysis with a first order oral absorption model with lag time and linear elimination from the first compartment was conducted to identify covariates influencing drug exposure. Inter-individual and inter-occasional variability were modeled for apparent oral clearance to study the effect of comedication on Ruxolitinib. The model was encoded in mrsgsolve for R and Monolix.

Main outcome measures: The impact of comedication, patient characteristics and laboratory parameters on Ruxolitinib plasma concentrations were investigated.

Results: Ruxolitinib exposure was evaluated in 23 patients (168 samples). Median Ruxolitinib trough concentration was $41.1 \mathrm{ng} / \mathrm{mL}$ at $20 \mathrm{mg}$ daily (IQR $40.7 \mathrm{ng} / \mathrm{mL}$, range $2.7-229 \mathrm{ng} / \mathrm{mL}$ ). Our population pharmacokinetic analysis revealed that concentrations in our cohort were significantly higher than in a population of patients with myelofibrosis $(\mathrm{p}<0.001)$, caused by a significant reduction in Ruxolitinib clearance by approximately $50 \%$. Additional comedication with at least one strong CYP3A4 or CYP2C9 inhibitor had a significant effect on Ruxolitinib clearance $(\mathrm{p}<0.001)$, leading to a further reduction by $23 \%$. No other covariate affected exposure significantly. Conclusion: In conclusion, our findings show that TDM of Ruxolitinib in GvHD patients might be beneficial for risk groups to ensure therapeutic success and reduction of adverse events.

Disclosure of Interest: F. Aghai: None Declared, N. Isberner Grant/ Research support from Hector Stiftung II, S. Zimmermann: None Declared, M. Kurlbaum: None Declared, S. Kraus Grant/Research support from Jazz Pharmaceuticals, medac GmbH, Janssen, BMS, Pfizer, G. U. Grigoleit: None Declared, O. Scherf-Clavel Grant/Research support from Hector Stiftung II, Horphag Ltd., H. Klinker Consultant for AbbVie, BMS, Gilead, Hexal, Janssen, MSD, Shionogi, ViiV, Grant/Research support from Hector Stiftung II, Abbvie, BMS, Gilead, Janssen, MSD.

\section{PP02}

Community pharmacists' perspectives of the barriers and enablers of their role to optimise opioids therapy for chronic non-malignant pain patients

\section{A. Alenezi ${ }^{1}$, V. Paudyal ${ }^{1, *}$, A. Yahyouche ${ }^{1}$}

${ }^{1}$ School of Pharmacy, Institute of Clinical Sciences, University of Birmingham, Birmingham, United Kingdom

Background and Objective: Opioids are widely used to manage chronic non-malignant pain, but there is growing concern about harms resulting from excessive and inappropriate use of prescription opioids. Community pharmacists have potential role in the optimisation of chronic opioid therapy.

Objective: The study seeks to identify facilitators and barriers of community pharmacists' potential role in optimising chronic opioid therapy for non-malignant pain.

Setting and Method: Setting The research was carried out in community pharmacies in several UK cities.

Method: Semi-structured interviews based on the Theoretical Domains Framework were conducted face to face and telephone, with 20 community pharmacists. Interviews were audio-recorded and transcribed verbatim, and data analysed deductively and inductively.
Excerpts were assigned to one or more frameworks 14 domains and, in addition, extra-domain subthemes identified. The relative relevance of domains was assessed based on frequency of related statements and pharmacists' emphasis on the impact of the factor concerned.

Main outcome measures: Main outcomes Pharmacists demonstrated awareness of opioid-related problems and identified potential for their enhanced role in mitigating the problems.

Results: Community pharmacits saw themselves as guardians of patient welfare, a role they performed through education and advice, monitoring (albeit limited) and referral of concerns to the prescribers. However, their role in opioid optimisations was challenged by environmental, systemic and personal factors. They lacked relevant training, time and resources, experienced difficulties arising from repeated ordering and prescription delivery system that reduced contact with patients and were constrained by lack of authority perceptions that opioid optimisation is the prescriber's responsibility and problem communication with doctors. They offered suggestions for alleviating the opioids epidemic by improving opioid prescribing, enhancing their own role through training and resources and changing patient behaviour via more effective education and monitoring.

Conclusion: The role of the community pharmacists in optimising chronic opioid therapy neither well defined nor implemented in the UK. Realisation of their potential contributions in this area should be part of integrated, patient -centred clinical practice, supported by clear consistent guidelines, training and resources.

Disclosure of Interest: None Declared.

\section{PP04}

Adherence to secondary prevention medications in patients with stroke in Saudi Arabia

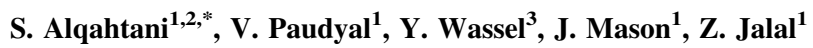

${ }^{1}$ Pharmacy college, University of Birmingham, birmingham, United Kingdom, ${ }^{2}$ Pharmacy college, Jazan University, Jazan, Saudi Arabia, ${ }^{3}$ Medicine College, Mansoura University, Mansoura, Egypt

Background and Objective: To assess stroke patients' adherence to secondary preventive medications in Saudi Arabia.

Setting and Method: A cross-sectional study was conducted in the Armed Forces Hospital in the Southern region of Saudi Arabia. The validated Arabic version of Hill-Bone Medication Adherence Scale (HB-MAS) was used.

Main outcome measures: Defining levels of adherence (optimal and suboptimal adherence) among stroke patients and examining the relationship between a set of predictors and adherence rate among such patients.

Results: A total of 354 stroke patients were invited and 343 (97\%) completed the questionnaire. The mean \pm SD age of participants was $67.8 \pm 13.57$ years; Only 95 patients $(27.7 \%)$ had optimal adherence with a high majority $248(82.3 \%)$ having suboptimal adherence to stroke secondary preventive medications (SSPM). Males (224) showed suboptimal adherence (75.9\%) more than females $(65.5 \%)$, which was statistically significant $X^{2}(\mathrm{df}=1, \mathrm{n}=343)=4.2$, $\mathrm{p}$-value $=0.04$. The average age was significantly higher in suboptimal group (69.65 years) compared to optimal group (64.33 years) and $U\left(\mathrm{~N}_{\text {optimal adherence }}=95, \mathrm{~N}_{\text {suboptimal adherence }}=248\right)=2.165$, $\mathrm{p}$-value $=0.03$. Binary regression analysis revealed that per participant, the number of strokes $(\mathrm{OR}=1.779,95 \% \mathrm{CI}: 2.03-17.30$, p-value $=0.001)$, and number of other medications used $(\mathrm{OR}=1.61$, 95\% CI: $1.26-2.05$, p-value $<0.001)$ were significantly associated with suboptimal adherence.

Conclusion: Overall, adherence to stroke secondary preventive medications (SSPM) was suboptimal among the study participants and associated with recurrent stroke. Evidence-based targeted 
interventions on medication adherence and adherence strategies should be established and implemented to improve treatment outcomes.

Disclosure of Interest: None Declared.

\section{PP05}

Patterns of potentially inappropriate hypnosedative use in Czech acute care seniors: outputs from the inomed and EUROAGEISM H2020 projects

O. Antonenko ${ }^{1, *}$, G. Vaculová ${ }^{1}$, A. Zelinková ${ }^{1}$, M. Halačová ${ }^{1}, S$. Grešáková $^{1}$, J. Reissigová ${ }^{2}$, D. Fialová ${ }^{1,3}$

${ }^{1}$ Department of Social and Clinical Pharmacy, Faculty of Pharmacy in Hradec Králové, Charles University, Hradec Králové, ${ }^{2}$ Department of Statistical Modeling, Institute of Computer Science of the Czech Academy of Sciences, ${ }^{3}$ Department of Geriatrics and Gerontology, 1st Faculty of Medicine, Charles University, Prague, Czech Republic

Background and Objective: Particularly older adults acutely hospitalized suffer from higher rates of insomnias and frequently use hypnosedative drugs. The aim of our study was to describe the prevalence of insomnia and patterns of inappropriate hypnosedative use in acutely hospitalized seniors in the Czech Republic.

Setting and Method: 438 older patients ( $\geq 65$ yrs) acutely hospitalized at 3 regionally different hospitals (on geriatric wards) have been assessed by comprehensive geriatric assessment using the EUROAGEISM H2020 study protocols. For identification of potentially inappropriate patterns of hypnosedatives use, the explicit geriatric criteria of potentially inappropriate medications (PIMs), mainly 2019 Beers criteria and 2015 EU(7)-PIM list were applied.

Main outcome measures: Insomnia (according to medical records) was diagnosed in $16.9 \%(\mathrm{~N}=74)$ of seniors, but $34,6 \%$ used hypnosedatives in the evening or at night doses $(\mathrm{e} / \mathrm{n}) .13 .8 \%$ of seniors reported use of drugs at e/n aggravating insomnias (beta-blockers, diuretics, theophylline). Among most frequently used hypnosedatives (e/n) were identified:antipsychotics(18.5\%), Z-drugs(16,2\%) and benzodiazepines (BZD, 14,2\%).

Results: Non-geriatric doses were determined in users of Z-drugs $(10.5 \%)$, sedative antidepressants e/n $(1,8 \%)$ and longer than recommended duration of geriatric drug therapy for Z-drugs (5.9\%, > 1 month), BZDs e/n $(5,3 \%,>1$ month $)$ and sedative antidepressants e/n $(3,3 \%,>6$ months). Combination of hypnosedatives was identified in $5.9 \%$ of patients.

Conclusion: Patterns of potentially inappropriate hypnosedative were documented particularly in frequent indication of antipsychotics $\mathrm{e} / \mathrm{n}$, inappropriate dosing of Z-drugs and long-term use of BZDs.

Grants: InoMed project (reg. No: CZ.02.1.01/0.0/0.0/18_069/ 0010046, 2019-2022), EUROAGEISM H2020-MCSF-ITN-764632 project, PROGRESS Q42, Faculty of Pharmacy, Charles University (KSKF-2 Assoc. Prof. Fialová) and SVV 260,417.

Disclosure of Interest: None Declared.

\section{PP06}

Appropriateness of hypnosedative drug use in seniors in ambulatory care in the Czech Republic: results from the InoMed and EUROAGEISM H2020 projects

O. Antonenko, ${ }^{1, *}$, G. Vaculová ${ }^{1}$, K. Puldová ${ }^{1}$, M. Halačová ${ }^{1}$, S. Grešáková $^{1}$, J. Reissigová ${ }^{2}$, D. Fialová ${ }^{1,3}$

${ }^{1}$ Department of Social and Clinical Pharmacy, Faculty of Pharmacy in Hradec Králové, Charles University, Hradec Králové, ${ }^{2}$ Department of Statistical Modeling, Institute of Computer Science of the Czech
Academy of Sciences, ${ }^{3}$ Department of Geriatrics and Gerontology, 1st Faculty of Medicine, Charles University, Prague, Czech Republic

Background and Objective: Hypnosedatives are one of the most often prescribed medications in ambulatory seniors due to high prevalence of insomnia in older patients. The aim of this study was to describe the prevalence of insomnia, hypnosedative drug use and inappropriate patterns of their prescribing (nongeriatric doses and nongeriatric lenght of therapy) in ambulatory care seniors in the Czech Republic.

Setting and Method: 563 ambulatory geriatric patients ( $\geq 65$ years) have been prospectively assessed at 4 regionally different geriatric ambulances (about 150 seniors per site). Comprehensive geriatric assessment using the EUROAGEISM H2020 study protocols was apllied. For the identification of potentially inappropriate prescribing patterns in hypnosedative drug use, 2019 Beers criteria and 2015 EU(7)-PIM criteria were applied.

Main outcome measures: $30.6 \%(\mathrm{~N}=172)$ of ambulatory seniors suffered from insomnias and the majority of them were treated only pharmacologically(24.0\%). Hypnosedatives in the evening or at night $(\mathrm{e} / \mathrm{n})$ were prescribed mainly in the age group of patients 84-94 years old $(42.8 \%)$.

Results: In prescription dominated Z-drugs(8.2\%), benzodiazepines(BZDs,7.6\%), from ...off-label "hypnotics antipsychotics (17.8\%, !17\% of users $>6$ months), sedative antidepressants $(9.2 \%)$. In nongeriatric doses and nongeriatric lenght of therapy were prescribed Z-drugs $(6.0 \%$ and $2,7 \%$, respectively), BZDs e/n $(2,7 \%$ and $11 \%)$ and above stated antipsychotics.

Conclusion: In ambulatory care seniors in the Czech Republic, nonpharmacological methods of treatment were rarely used and hypnosedative drugs were extensively prescribed particularly in cohort of very old patients $(85+)$.

Grants: InoMed project (reg. No: CZ.02.1.01/0.0/0.0/18_069/ 0010046, 2019-2022), EUROAGEISM H2020-MCSF-ITN-764632 project, PROGRESS Q42, Faculty of Pharmacy, Charles University (KSKF-2 Assoc. Prof. Fialová) and SVV 260417 program.

Disclosure of Interest: None Declared.

\section{PP07}

Ageing in developing countries and appropriateness of geriatric prescribing: physicians' knowledge of the explicit criteria of potentially inappropriate medications

D. K. Bandari ${ }^{1, *}$, A. S. Bhagavathula ${ }^{1}$, B. S. Areman ${ }^{1}$, M. Chhabra $^{2}$, J. Brkic ${ }^{1}$, J. Reissigová ${ }^{3}$, D. Fialova ${ }^{1,4}$

${ }^{1}$ Department of Social and Clinical Pharmacy, Faculty of Pharmacy in Hradec Kralove, Prague, Czech Republic, ${ }^{2}$ Department of Pharmacy Practice, Indo Soviet Friendship College of Pharmacy, Punjab, India, ${ }^{3}$ Department of Statistical Modeling, Institute of Computer Science of the Czech Academy of Sciences, ${ }^{4}$ Department of Geriatrics and Gerontology, 1st Faculty of Medicine, Charles University, Prague, Czech Republic

Background and Objective: Potentially Inappropriate Medication (PIM) prescribing is associated with increased mortality and morbidity. Prescribing patterns are strongly influenced by physicians' knowledge and expertise in geriatric prescribing. We assessed physician's knowledge of specific explicit criteria of PIMs, usage of different information sources and guidelines, and barriers among physicians in their daily clinical practice.

Setting and Method: Descriptive cross-sectional study among physicians $(\mathrm{N}=256)$ in two tertiary care teaching hospitals was conducted. Physicians' geriatric knowledge, characteristics, use of general and geriatric guidelines and physicians' barriers to appropriate geriatric prescribing was assessed using validated 
questionnaire. Pearson's Chi-square test, Student's t-test, MannWhitney's U test and logistic regression model were conducted to determine main characteristics and influence of variables on appropriateness prescribing decisions. $\mathrm{R}$ software (version 4.0.3) was used for data analysis, p-value $<0.05$ was considered statistically significant.

Main outcome measures: Physician's knowledge on explicit criteria of PIM's, usage of different guidelines and barriers towards appropriate geriatric prescribing were analyzed using descriptive statistics and multivariate logistic regression models.

Results: Of 201 respondents (78.5\% response rate), majority were males $(63.2 \%)$, mean age $33.3 \pm 6.3$ years. Three-fourths $(74.1 \%)$ received training in geriatric medicine, $39.8 \%$ were currently providing more than once a week care for older adults in long-term care facilities and one third (32.3\%) were providing acute care. However, only $31.8 \%$ physicians felt confident in prescribing older patients. Mean score of clinical vignettes was $3.5 \pm 0.9$ (scale: $\min 0$-max 5). Multiple logistic regression model confirmed better geriatric knowledge in females $(\mathrm{OR}=2.3,95 \%$ CI 1.0-5.2), age group of $30-39$ years $(\mathrm{OR}=4.6,95 \% \mathrm{CI} 2.0-10.8)$ compared to 22-29 years, physicians providing geriatric care in long-term care facilities less than once weekly $(\mathrm{OR}=4.8,95 \% \mathrm{CI} 1.6-14.9)$ compared with those not providing geriatric care. The odds of better geriatric knowledge was also significantly associated with the extend of use of explicit PIM criteria and physician specialties.

Conclusion: Inadequate knowledge and low confidence towards appropriate geriatric prescribing was reported among study participants. Educational interventions and integrative training in rational geriatric pharmacotherapy may improve skills of physicians in appropriate prescribing to older patients. Received funding from the INOMED project (CZ.02.1.01/0.0/0.0/18_069/0010046), EUROAGEISM H2020-MCSF-ITN-764632 project, PROGRESS Q42, Faculty of Pharmacy, Charles University (KSKF-2 Assoc. Prof. Fialova) and SVV 260417.

Disclosure of Interest: None Declared.

\section{PP09}

Prevalence of polypharmacy and risk of potentially inappropriate medication use in the older population in a developing country: a systematic review and meta-analysis

\section{A. S. Bhagavathula ${ }^{1, *}$, D. Fialova ${ }^{1,2}$}

${ }^{1}$ Social and Clinical Pharmacy, Faculty of Pharmacy in Hradec Králové, Charles University, Hradec Kralova, ${ }^{2}$ Geriatrics and Gerontology, 1 st Faculty of Medicine, Charles University, Prague, Czech Republic

Background and Objective: Polypharmacy and potentially inappropriate medication (PIM) use in older populations $(65+$ years) have not yet been investigated by meta-analyses in developing countries. This systematic literature review and meta-analysis aimed to investigate the prevalence of polypharmacy and PIM use and major risk factors associated with PIM prescribing in older adults in Ethiopia.

Setting and Method: We searched PubMed/MEDLINE, Scopus, Embase, and Google Scholar databases to identify relevant studies published between January 1990 to October 2020. Observational studies reporting the prevalence and association of risk factors with polypharmacy and PIM use in the older population were meta-analyzed. A multilevel meta-analysis was conducted to pool the prevalence estimates, and the risk of PIM use was reported as a relative risk (RR) with a $95 \%$ confidence interval (CI).

Main outcome measures: To assess the prevalence of polypharmacy and PIM use.
Identify the factors associted with increased risk of PIM use in Ethiopian older population.

Results: We identified by systematic literature review 404 articles. Of those, eight studies fulfilled inclusion criteria, comprising a total sample of 2608 participants. The overall prevalence of polypharmacy and PIM use pooled by meta-analysis in the Ethiopian older population was $33 \%$ and $37 \%$, respectively. The risk factors of PIM use were analyzed in the meta-analysis (particularly polymorbidity, polypharmacy, gender, and older age), and only older age of $65+$ (RR:1.71, 95\% CI: 1.16 - 2.51) was significantly associated with PIM use.

Conclusion: This first meta-analysis from a developing country revealed a high prevalence of polypharmacy and PIM use in the Ethiopian older population. There was no awareness about the risk of PIMs in patients with polypharmacy and polymorbidity, and older age significantly predicted PIM use. Interventions ensuring rational geriatric pharmacotherapy are essential in developing countries in order to reduce the expected burden of PIM-related geriatric morbidity, higher costs, and mortality.

InoMed project (reg. No: CZ.02.1.01/0.0/0.0/18_069/0010046, 2019-2022), EUROAGEISM H2020-MCSF-ITN-764632 project, PROGRESS Q42, Faculty of Pharmacy, Charles University (KSKF-2 Assoc. Prof. Fialová) and SVV 260417.

Disclosure of Interest: None Declared.

\section{PP10}

Prevalence of polypharmacy, hyperpolypharmacy and potentially inappropriate medication use in the older population in India: a systematic review and meta-analysis of 27 observational studies

A. S. Bhagavathula ${ }^{1, *}$, K. Vidyasagar ${ }^{2}$, M. Chhabra ${ }^{3}$, M. Rashid ${ }^{4}$, R. Sharma ${ }^{3}$, D. K. Bandari ${ }^{1}$, D. Fialova ${ }^{1,5}$

${ }^{1}$ Social and Clinical Pharmacy, Faculty of Pharmacy in Hradec Králové, Charles University, Hradec Kralova, Czech Republic,

${ }^{2}$ Pharmaceutical Sciences, University College of Pharmaceutical Sciences, Warangal, ${ }^{3}$ Pharmacy Practice, Indo-Soviet Friendship College of Pharmacy, Moga, ${ }^{4}$ Pharmacy Practice, Sri

Adichunchanagiri College of Pharmacy, Banglore, India,

${ }^{5}$ Department of Geriatrics and Gerontology, 1 st Faculty of Medicine, Charles University, Prague, Czech Republic

Background and Objective: There is limited evidence of the prevalence of polypharmacy, hyperpolypharmacy, and potentially inappropriate medication (PIM) use in older people in developing countries. We investigated regional variations in the prevalence of polypharmacy, hyperpolypharmacy, and PIM use in older people $(60+$ years $)$ in India.

Design: Studies were identified through Medline/PubMed, Embase, Scopus, and Google Scholar databases published from inception (2002) to September 31, 2020. Of the total 2000 articles, 27 were included.

Results: Overall, the pooled prevalence of polypharmacy $(49 \%, 95 \%$ confidence interval: 42-56), hyperpolypharmacy $(31 \%, 21-40)$, and PIM use in the older population in India was 28\% (24-32). Polypharmacy was more prevalent in North India (72\%, 64-79), and hyperpolypharmacy was frequently observed in inpatient care $(37 \%$, 26-47). Regional estimates for the pooled prevalence of PIM use were as follows: $23 \%(21-25)$ for East, West $(33 \%, 24-42)$, North $(17.8 \%, 11-23)$ and $32 \%(26-38)$ in South India; $31 \%(24-38)$ was observed in older inpatients, and 27\% (18-35\%) in private hospitals. Overall estimates of the prevalence of PIM use in the older population aged $\geq 70$ was $35 \%$ (28-42), females $34 \%$ (22-46), and a higher number of PIMs ( $\geq 3$ ) 29\% (22-36). 
Conclusion: In India, polypharmacy and hyperpolypharmacy are widely prevalent, and $28 \%$ of the older people are affected by PIM use. Thus, appropriate steps are needed to promote rational geriatric prescribing in India.

InoMed project (reg. No: CZ.02.1.01/0.0/0.0/18_069/0010046, 2019-2022), EUROAGEISM H2020-MCSF-ITN-764632 project, PROGRESS Q42, Faculty of Pharmacy, Charles University (KSKF-2 Assoc. Prof. Fialová) and SVV 260,417.

Disclosure of Interest: None Declared.

\section{PP11}

Potentially inappropriate prescribing in older adults in central and Eastern Europe: systematic literature review

J. Brkic ${ }^{1, *}$, B. Okuyan ${ }^{2}$, I. Kummer ${ }^{1}$, J. Reissigova ${ }^{3}$, S. Sesto ${ }^{4}$, A. Capiau $^{5,6}$, M. Ortner Hadžiabdić ${ }^{7}$, I. Tadic ${ }^{4}$, K. Tachkov ${ }^{8}$, V. Bobrova', D. Fialova ${ }^{1,10}$

${ }^{1}$ Department of Social and Clinical Pharmacy, Faculty of Pharmacy in Hradec Kralove, Charles University, Hradec Kralove, Czech Republic, ${ }^{2}$ Clinical Pharmacy Department, Faculty of Pharmacy, Marmara University, Istanbul, Turkey, ${ }^{3}$ Department of Statistical Modelling, Institute of Computer Science, Czech Academy of Sciences, Prague, Czech Republic, ${ }^{4}$ Department of Social Pharmacy and Pharmaceutical Legislation, Faculty of Pharmacy, University of Belgrade, Belgrade, Serbia, ${ }^{5}$ Department of Pharmacy, Ghent University Hospital, ${ }^{6}$ Pharmaceutical Care Unit, Faculty of Pharmaceutical Sciences, Ghent University, Ghent, Belgium, ${ }^{7}$ Centre for Applied Pharmacy, Faculty of Pharmacy and Biochemistry, University of Zagreb, Zagreb, Croatia, ${ }^{8}$ Department of Social Pharmacy and Pharmacoeconomic, Medical University of Sofia, Sofia, Bulgaria, ${ }^{9}$ Institute of Pharmacy, Faculty of Medicine, University of Tartu, Tartu, Estonia, ${ }^{10}$ Department of Geriatrics and Gerontology, 1st Faculty of Medicine, Prague, Czech Republic

Background and Objective: Potentially inappropriate prescribing (PIP) in older adults is a serious issue associated with adverse health outcomes. Our aim was to systematically review the literature on the prevalence of PIP in older adults in Central and Eastern Europe (CEE).

Setting and Method: We searched two electronic databases MEDLINE and Embase in June 2019 and reference lists of included studies and relevant reviews. We included studies that assessed the prevalence of PIP in older adults (aged 60 years and older) in all settings of care using validated tools. At least two authors independently selected studies, extracted data, assessed risk of bias (using Joanna Briggs Institute's critical appraisal checklist for studies reporting prevalence data), and assessed certainty of evidence (according to GRADE approach). Meta-analysis was not appropriate due to large variations in outcome measurement across studies. Thus, we synthesized data using descriptive statistics-median, range and interquartile range.

Main outcome measures: Prevalence of PIP in older adults.

Results: Our search yielded 1890 records, of which we included 27 studies (28 records). The design of all studies was cross-sectional, except for one study that was uncontrolled before-after. Most studies were conducted in high-income countries of European Union 11 (EU11). Only explicit tools were used to measure PIP prevalence. The prevalence of PIP was: median $34.6 \%$, minimum $6.5 \%$, maximum $95.8 \%$, interquartile range $25.9-63.2 \%$; 26 studies, $1,139,693$ participants; very low certainty of evidence. The median PIP prevalence was higher in studies conducted in long-term and outpatient care than in studies conducted in acute and community care $(71.1$ and $53.8 \%$ vs 34.5 and $31.5 \%$, respectively). Surprisingly, the prevalence of PIP was higher in studies conducted after 2010 than before 2010 (55.0 vs $25.7 \%$ ).

Conclusion: This review has shown that PIP in older adults is prevalent in CEE countries. Further well-designed and well-reported cross-national studies need to be carried out.

Grant support of research group: European Union's Horizon 2020 research and innovation progamme under the Marie SkłodowskaCurie grant agreement No 764632. PROGRESS Q42, Faculty of Pharmacy, Charles University (KSKF-2 Assoc. Prof. Fialova), SVV 260417 and INOMED project (CZ.02.1.01/0.0/0.0/18_069/0010046). Disclosure of Interest: None Declared.

\section{PP12}

Cost saving analysis after the introduction of adalimumab biosimilars in an Italian hospital

G. De Vivo ${ }^{1, *}$, E. Calzavara ${ }^{1}$, S. Nobili ${ }^{1}$, L. Gambitta ${ }^{1}$, E. Magni ${ }^{1}$, S. Vimercati ${ }^{1}$

${ }^{1}$ ASST Fatebenefratelli-Sacco, Milan, Italy

Background and Objective: Inflammatory bowel disease (IBD) is a chronic and disabling disease characterized by a disproportionate inflammatory response in the gut, tissue damage and malabsorption. Currently, adalimumab, in our Hospital, is mostly prescribed to patients with IBD refractory to corticosteroids and immunosuppressant drugs. Since on one hand adalimumab originator (ADA-O) is more expensive than adalimumab biosimilars (ADA-B) and on the other IBD prevalence is increasing steadily, Hospital Pharmacists have been sensitizing clinicians towards ADA-B, since its introduction in 2019. Moreover, the Italian Authority of Drugs Position Paper on biosimilars, states that all new patients should start the therapy with the cheapest biosimilar. The objective is to compare the adalimumab expenditure and savings after the introduction of ADA-B, between the years 2019 and 2020 .

Design: A cost analysis was conducted by the Pharmacy of a Community Hospital in the north of Italy over the period January 2019December 2020, by extracting prescription data from the Pharmacy management software in an excel database used to summarize data thanks to descriptive statistic methods. To perform the analysis, the real costs spent in overall ADA prescriptions (ADA-O + ADA-B) were compared to the hypothetical costs if only ADA-O had been prescribed.

Results: In 2019, the hypothetical costs of ADA-O prescriptions would have been of $€ 317,976.96$ (1888 pens, average price of $€ 168.42 /$ each), compared to the real ones of $€ 253,997.99$ (1234 ADA$\mathrm{O}$ pens, 654 ADA-B pens with different costs). The introduction of ADA-B led to a consequent saving of $€ 63,978.96$ (-20\%).

In 2020, if only ADA-O had been prescribed, the costs would have raised by $15 \%(+€ 55.253 .04)$ leading to a total amount of $€ 373,230.00$ ( 2262 pens), due to an increase of patients by $26 \%$. Since the real cost was of $€ 215,533.89$ (450 ADA-O pens and 1812 ADA-B pens), the increased prescription of ADA-B led to a saving of $€ 157,696.11$ ( $-42 \%)$.

Conclusion: The present analysis suggests that the increasing ADA-B prescription over the period January 2019-December 2020 led to an overall cost saving of $€ 221.675,08$. Hospital Pharmacists, by their counselling work, are essential to sensitize clinicians towards the use of biosimilars thus ensuring the financial sustainability of National Health System.

Disclosure of Interest: None Declared. 


\section{PP13}

Drug burden index in older community dwelling patients in Croatia: preliminary results from the Croatian study sample of the EUROAGEISM ESR7 H2020 project

\section{Držaicic ${ }^{1, *}$, I. Kummer ${ }^{1,2}$, I. Bužančić ${ }^{1}$, M. Marušić ${ }^{3}$, M.} Radovanlija $^{4}$, S. Falamić ${ }^{5}$, J. Brkić ${ }^{2}$, M. Ortner Hadžiabdićc ${ }^{6}$ D. Fialová ${ }^{2,7}$

${ }^{1}$ City Pharmacies Zagreb, Zagreb, Croatia, ${ }^{2}$ Department of Social and Clinical Pharmacy, Faculty of Pharmacy in Hradec Králové, Charles University, Hradec Králové, Czech Republic, ${ }^{3}$ Community Pharmacy Sonja Grbac Stublić, Zagreb, ${ }^{4}$ Community Pharmacy chain Rajić, Požega, ${ }^{5}$ Community Pharmacy Branka Marušić, Donji Miholjac, ${ }^{6}$ Faculty of Pharmacy and Biochemistry, University of Zagreb, Zagreb, Croatia, ${ }^{7}$ Department of Geriatrics and Gerontology, 1st Faculty of Medicine, Charles University in Prague, Prague, Czech Republic

Background and Objective: Aging population is at increased risk of negative health outcomes due to the use of potentially inappropriate medications. This study aimed to evaluate the anticholinergic and sedative medication burden and its correlation with patients' sociodemographic and health-related characteristics in older patients. Setting and Method: Data for this observational, cross-sectional study were collected using the EUROAGEISM H2020 ESR 7 project protocol in three community pharmacies in Zagreb. Presented data are preliminary and include 84 adults aged $65+$ years who were able and willing to give their informed consent. Collected data were analysed using IBM SPSS v 20.

Main outcome measures: Drug Burden Index (DBI); clinical and functional factors (hospitalizations, falls and emergency department visits during last year, mobility and strength, frailty score, number of used medications and self-reported health).

Results: 84 respondents were included in the study $(67.9 \%$ female; median age 73 (interquartile range 68-80)). Polypharmacy $(5+$ medications) was identified in $67.8 \%$ patients and $51.2 \%$ used $1+$ medication from DBI list. Women were prescribed DBI medications more often $(59,6 \%$ vs $33,3 \% ; \mathrm{p}=0,024)$ and had DBI index higher than men $(0.62 \pm 0.72$ vs $0.24 \pm 0.44)(\mathrm{p}=0,004)$. The most commonly prescribed DBI drug classes were anxiolytics $(58.6 \%)$, mostly benzodiazepines, followed by antidepressants and analgesics. The most prevalent were sedative DBI medications; diazepam $22.6 \%$, alprazolam $13.1 \%$, tramadol $8.3 \%$, zolpidem $6.0 \%$, respectively, while the only anticholinergic medication was trospium, prescribed to $3.6 \%$ of patients. A positive correlation was observed between DBI index and the number of prescribed medications and frailty score ( $\rho=0.51, p<0.001 ; \rho=0.26, p=0.019$, respectively), while correlation found between DBI index and self-reported health was negative $(\rho=-0.32, p=0.003)$.

Conclusion: Higher DBI was found in women and participants with polypharmacy. Patients with higher DBI scored higher on frailty test, and reported their health status as worse. These findings indicate additional attention is needed when providing care to these patients and warrants considering the need for potential deprescribing.

Grant support: the EUROAGEISM H2020-MCSF-ITN-764632 project, INOMED project (CZ.02.1.01/0.0/0.0/18_069/0010046), PROGRESS Q42, Faculty of Pharmacy, Charles University (KSKF-2 Assoc. Prof. Fialova) and SVV 260,417.

Disclosure of Interest: None Declared.

\section{PP15}

Case report: effectiveness and safety of Vedolizumab dose interval reduction strategy in inflamatory bowel disease

D. Guerra Estévez ${ }^{1, *}$, C. Palomo Palomo ${ }^{1}$, M. M. Romero Alonso $^{1}$, J. Estaire Gutierrez ${ }^{1}$

${ }^{1}$ Pharmacy, Hospital Infanta Elena, Huelva, Spain

Background and Objective: Vedolizumab is an option in the treatment of Inflammatory Bowel Disease (IBD), including Ulcerative Colitis (UC) and Crohn's Disease (CD). The objective of this study is to evaluate the effectiveness and safety of Vedolizumab at standard and intensified doses in the treatment of IBD.

Setting and Method: Retrospective observational study (May 2016December 2020) in which patients with IBD treated with vedolizumab for at least 20 weeks were included. Demographic, clinical, analytical and pharmacotherapeutic variables were collected.

Main outcome measures: Effectiveness was evaluated by the normalization of C-Reactive Protein (CRP) and Fecal Calprotectin (FCT) during treatment, the need for dose intensification(DI) and the clinical assessment reported by the attending physician. Safety was assessed by documented adverse events (AE). DI was defined as the shortening of the dose interval from 8 to 4 weeks.

Results: 21 patients (13 men) with a median age of 43(15-68)years were included, 15 diagnosed with CD and 6 with UC. 15 patients had prior treatment with $\geq 2$ anti-tumor necrosis factor (anti-TNF) drugs, 5 with one, and one patient with Ustekinumab. 15 patients received concomitant treatment with immunosuppressants. The median duration of treatment was 72 (24-212) weeks. In 10 patients the treatment was suspended due to loss of efficacy with a median of $60(28-104)$ weeks; being realized DI in all with a median of 30 (12-80)weeks. At the time of the study, 10 patients continued in treatment with good clinical response, with a median treatment time of 102 (32-212) weeks. In these patients the dose having been intensified in 3 (at weeks 10, 44 and 84). Before treatment with vedolizumab, the CRP and FCT values were high in 14 and 20 patients, respectively. During treatment, CRP was normalized in 4 patients and FCT in 2. One patient discontinued Vedolizumab at 24 weeks by AE (genital and oral herpes), another patient presented paresthesias in hands, which were decreasing in successive administrations, and another presented swelling in the legs.

Conclusion: Vedolizumab has a moderate effectiveness and safety in the treatment of IBD. However, due to the few current therapeutic options, we can consider it as another alternative. Compared to other studies, in our group of patients the dose intensification strategy has not been able to increase the effectiveness of the drug.

Disclosure of Interest: None Declared.

\section{PP16}

General disorders induced by intravesical immunotherapy of BCG Danish strain 1331

D. Guerra Estevez ${ }^{1, *}$, M. M. Romero Alonso ${ }^{1}$, C. Palomo Palomo $^{1}$, J. Estaire Gutierrez ${ }^{1}$

${ }^{1}$ Pharmacy, Hospital Infanta Elena, Huelva, Spain

Background and Objective: Intravesical instillation of BCG has been shown to reduce recurrences of non-muscle invasive bladder 
cancer (NMIBC). For the induction phase, the BCG available in Spain is the Tice strain (TS), while for maintenance phase, we have the Danish strain 1331 (DS1331), which must be requested through the Spanish Agency for Medicines and Health Products (AEMPS). Common general disorders $(\geq 1 / 100$ to $<1 / 10)$ of intravesical immunotherapy with BCG DS1331 include malaise, subfebrile, and influenza-like symptoms. The objetive is to assess the general disorders induced by intravesical immunotherapy of BCG DS1331.

Setting and Method: Retrospective study in which 15 patients with high-grade NMIBC were included. All had previously completed induction treatment with BCG TS (6 weekly instillations) and received the first maintenance dose with BCG DS1331 between August 2018 and December 2020.

Main outcome measures: The variables analyzed were age, clinical history and comorbidities, and appearance of systemic adverse reactions.

Results: 15 patients ( 3 women) were included, with a median age of 70 (39-85). 14 had comorbidities, the most common being arterial hypertension (11), dyslipidemia (9) and diabetes mellitus (5). After the first administration of BCG DS1331, 6 patients with a median age of 68.5 (62-65) influenza-like symptoms and expressed their intention to suspend treatment. No adverse reactions appeared during the previous induction phase with BCG CT, so it was decided to continue administering this strain also in the maintenance phase, without reappearing the general adverse reactions that they manifested with BCG DS1331.

Conclusion: According to our results, general disorders associated with intravesical immunotherapy with BCG DS1331 are very common adverse effects, since they have affected $\geq 1 / 10$ patients, not coinciding with the technical data sheet of the product. Furthermore, we can conclude that BCG DS1331 has a worse safety profile than BCG TS. We did not find a relationship between age or comorbidities and tolerability of BCG DS1331. However, a study that includes a greater number of patients is necessary to confirm our results.

Disclosure of Interest: None Declared.

\section{PP18}

\section{Use of anxiolytics during the COVID-19 pandemic}

\section{S. Kellici ${ }^{1, *}$, K. Kaja ${ }^{1}$}

${ }^{1}$ Pharmaceutical Department, University of Medicine, Tirana, Tirana, Albania

Background and Objective: The COVID-19 pandemic influenced significantly the people's lives worldwide. Concerns raised for the mental health, too. The pharmaceutic workforce was continuously involved in the pharmaceutical care service, without interrupting the service, even during the national lockdown. We tried to verify if there was any change in the use of anxiolytic drugs in the main Albanian city, Tirana; since the onset of the COVID-19 pandemic.

Setting and Method: A cross-sectional study was conducted in Tirana, the capital city of Albania during September 2020. The study was based on the interaction with 120 pharmacists of the community pharmacies. They were asked to fulfil a structured questionnaire, which included data on age, gender, years of professional experience of the pharmacists, use of anxiolytics among patients during the COVID-19 pandemic, characteristics of the anxiolytics' users and respective pharmaceutical care services.

Main outcome measures: Evidence the level of use of anxiolytic drugs.

Results: Within the sample of 120 pharmacists included in the study, most of them were females (75\%), aged 25-35 years, with less than 10 years of professional experience $(85 \%)$. $61 \%$ of the overall included pharmacists declared that they noticed an increase in the use of anxiolytics during the COVID-19 pandemic (March-May 2020). $22 \%$ of the pharmacists declare a further increase of anxiolytics' use even after May 2020. Anxiolytics' users are mainly females (65\%), aged 26-40 years (39\%). 76\% of the anxiolytics' users received other medications such as antidepressants or sleeping pills. $54 \%$ of the patients tried to receive the anxiolytic medications without a medical prescription. Only $40 \%$ of the patients having a medical prescription required pharmaceutical care assistance to the pharmacists.

Conclusion: This study provides a small evidence on the use of anxiolytics during the COVID-19 pandemic in Albania. We evidenced an increase in the use of these drugs, that is probably related to the pandemic. Further research is needed to confirm our results and to explore the factors contributing to this high level of use of anxiolytics. Disclosure of Interest: None Declared.

\section{PP19}

Drug-disease interactions' prevalence in nursing homes in the Czech Republic: findings from the InoMed and EU shelter projects

\section{Kummer ${ }^{1, *}$, A. Lukačišinová ${ }^{1}$, J. Brkić ${ }^{1}$, V. Př́íhodová ${ }^{1}$, J.} Reissigová $^{2}$, D. Fialová ${ }^{1,3}$

${ }^{1}$ Department of Social and Clinical Pharmacy, Faculty of Pharmacy in Hradec Králové, Charles University in Prague, Hradec Králové,

${ }^{2}$ Department of Statistical Modeling, Institute of Computer Science of the Czech Academy of Sciences, ${ }^{3}$ Department of Geriatrics and Gerontology, 1st Faculty of Medicine, Charles University in Prague, Prague, Czech Republic

Background and Objective: Potentially inappropriate medications (PIMs), non-geriatric dosing and drug-disease interactions (DDIs) in multimorbid, frail older patients can contribute to higher occurrence of adverse drug events and geriatric syndromes. This study aimed to identify the prevalence and specific features of DDIs prescribed in nursing home $(\mathrm{NH})$ residents in the Czech Republic.

Setting and Method: Our retrospective cross-sectional study analysed semi-implicitly (by retrospective implicit evaluation by clinical pharmacists) the InterRAI-LTCF study protocols of $490 \mathrm{NH}$ residents collected in 10 Czech $\mathrm{NH}$ facilities $(\mathrm{N}=490$, patients $65+$ ) participating in the EU SHELTER project (2009-2014). Retrospective evaluations of protocols were conducted in 2019 and statistical analyses in 2020 .

Main outcome measures: Total prevalence and prevalence of individual DDIs according to different sets of criteria, Beers 2012 criteria, Czech expert panel national consensus 2013 (CNC), and STOPP/ START criteria (version 1).

Results: The majority of participants were women (55.3\%); the mean age was $82.1 \pm 7.7$ years. Polypharmacy $(5+$ medications $)$ was identified in $58.0 \%$ of patients and $66.9 \%$ suffered from polymorbidity ( $5+$ diseases). Prevalence of at least 1 potential DDI using particular explicit criteria ranged from $44.5 \%$ to $62.3 \%$. (STOPP to $\mathrm{CNC}$ ). The most common DDIs in total sample were: use of benzodiazepines in depressive residents $(7.8 \%)$ and the use of opioids in residents with chronic constipation without co-treatment by osmotic laxatives $(7.4 \%)$. The prevalence of potential undertreatment (identified by START criteria) was $52.9 \%$, mainly due to absence of statins in patients suffering from type II DM and/or having relevant cardiovascular risk factors $(9.8 \%)$ and the absence of anticoagulation therapy in patients suffering from atrial fibrillation (7.1\%).

Conclusion: Potentially DDIs were highly prevalent in Czech $\mathrm{NH}$ seniors identified by application of different explicit criteria via method of retrospective semi-implicit reviews. The role of clinical pharmacists in NH setting is crucial in order to improve drug safety and appropriateness of drug prescribing in geriatric patients. 
Grants: InoMed project (reg. No: CZ.02.1.01/0.0/0.0/18_069/ 0010046, 2019-2022), EUROAGEISM H2020-MCSF-ITN-764632 project, SHELTER (FP7-HEALTH) project, PROGRESS Q42, Faculty of Pharmacy, Charles University (KSKF-2 Assoc. Prof. Fialová) and SVV 260,417.

Disclosure of Interest: None Declared.

\section{PP20}

CVS drug-disease interactions, the prevalence and risk factors in the Czech Republic: results from the InoMed and EUROAGEISM H2020 projects

I. Kummer ${ }^{1, *}$, J. Brkić ${ }^{1}$, A. Lukačišinová ${ }^{1}$, J. Reissigová ${ }^{2}$, D. Fialová ${ }^{1,3}$

${ }^{1}$ Department of Social and Clinical Pharmacy, Faculty of Pharmacy in Hradec Králové, Charles University, Hradec Králové, ${ }^{2}$ Department of Statistical Modeling, Institute of Computer Science of the Czech Academy of Sciences, ${ }^{3}$ Department of Geriatrics and Gerontology, 1st Faculty of Medicine, Prague, Czech Republic

Background and Objective: Cardiovascular (CVS) medications are the most frequently prescribed medications. Age-related pharmacological changes, polymorbidity and polypharmacy in seniors contribute to higher occurrence of drug-drug interactions, drug-disease interactions (DDIs) and adverse drug events. This study aimed to identify the prevalence and risk factors of CVS DDIs in ambulatory and acute care older patients.

Setting and Method: Data of 1,152 patients $65+$ were prospectively collected using the EUROAGEISM H2020 protocols in 4 acute care hospitals and 4 geriatric ambulances in the Czech Republic (Jun2019Jan2020, each study site $>150$ patients). Descriptive statistics and multiple logistic regression models was applied using R-software (version 4.0.3).

Main outcome measures: Prevalence and risk factors of CVS DDIs identified by different sets of criteria (STOPP/START criteria, Beers 2019 criteria and EU (7) PIM criteria). This abstract presents preliminary findings.

Results: The majority of older patients were women $(67.4 \%)$, and $38.7 \%$ were 85 + years old. Polypharmacy was identified in $85.3 \%$ of patients and $90.0 \%$ suffered from polymorbidity. The overall prevalence of CVS DDI was $88.3 \%$ (in acute and ambulatory care- $90.0 \%$ and $86.4 \%$, respectively $(\mathrm{p}=0.08)$ ). Based on individual criteria, the prevalence of DDIs was: START criteria $75.4 \%$, STOPP criteria $30.7 \%$, EU (7) PIM criteria $29.0 \%$ and BEERS criteria 21.9\%. Main risk factors associated with prescribing of CVS DDIs were: presence of $2+$ CVS diagnoses (Odds Ratio [OR] 12.243, 95\% Confidence Interval $[\mathrm{CI}]$ 7.298-21.452; $\mathrm{p}<0.001), 1+\mathrm{CVS}$ risk increasing diagnose (OR 3.276, 95\% CI 2.019-5.417; p < 0.001), age of $85+$ years (OR 3.143, 95\%CI 1.648-6.089; $\mathrm{p}<0.001)$; and polypharmacy (OR 2.227, 95\% CI 1.263-3.902; $\mathrm{p}=0.005$ ).

Conclusion: Our study confirmed high prevalence of CVS DDIs in Czech seniors in acute and ambulatory care. The risk of being prescribed CVS DDIs increased with polymorbidity, polypharmacy, higher prevalence of CVS disorders and disorder aggravating CVS risk. Regular medication check provided by trained clinical pharmacists should be promoted for older patients in different settings of care.

Grants: InoMed project (reg. No: CZ.02.1.01/0.0/0.0/18_069/ 0010046, 2019-2022), EUROAGEISM H2020-MCSF-ITN-764632 project, SHELTER (FP7-HEALTH) project, PROGRESS Q42, Faculty of Pharmacy, Charles University (KSKF-2 Assoc. Prof. Fialová) and SVV 260,417.

Disclosure of Interest: None Declared.

\section{PP21}

Use of Cladribine for multiple sclerosis in a second level hospital

L. Losa Lopez ${ }^{1, *}$, A. Murgadella Sancho ${ }^{1}$, B. Gracia Garcia ${ }^{1}$, A. Puebla Villaescusa $^{1}$, I. Zubizarreta Nafarrate ${ }^{2}$, E. Moral Torres ${ }^{2}$, E. Hidalgo Albert ${ }^{1}$

${ }^{1}$ Pharmacy, ${ }^{2}$ Neurology, Hospital Moisès Broggi, SANT JOAN DESPI, Spain

Background and Objective: The multiple sclerosis (MS) is the most common immune-mediated inflammatory demyelinating disease of the central nervous system. The pharmacist plays an important role in patient care due to the importance of pharmacological treatment. Cladribine is a new drug indicated for the treatment of patients with highly active relapsing MS. Objective: To assess the effectiveness and safety of cladribine in real clinical practice in a second level hospital. Design: Retrospective observational study with relapsing-remitting multiple sclerosis (RRMS) patients treated with cladribine (November 2018-December 2020). Pharmaceutical care was done during the dispensing of treatment. Data was collected through the electronic medical record and processed by Excel. The database included demographic variables, median time disease of MS, previous diseasemodifying therapy (DMT), doses of oral cladribine $(3.5 \mathrm{mg} / \mathrm{kg}$ of body weight divided into two yearly treatment courses $(1.75 \mathrm{mg} / \mathrm{kg}$ per treatment course) and adherence. To asses effectiveness, we analysed the adjusted annualized relapse rate (ARR) before versus ARR after the first dose of cladribine and neurological progression according to the Expanded Disability Status Scale (EDSS). To asses safety, a description of side effects was carried out according to Common Terminology Criteria for Adverse Events (v5.0).

Results: 16 patients, women(69\%), median age 44, median time disease of MS:10 years and median baseline EDSS:2.5. 15 patients were received previous DMT: fingolimod $(25 \%)$, glatiramer $(25 \%)$, interferon $(13 \%)$, natalizumab (13\%), teriflunomide $(12 \%)$ and dimethylfumarate $(6 \%) .1$ patient received the first course of treatment $(1.75 \mathrm{mg} / \mathrm{kg})$ and 14 patients completed second course of treatment $(3.5 \mathrm{mg} / \mathrm{kg})$ and the adherence was $100 \%$. The ARR before versus after cladribine administration was 0.56 (SD:0.51) and 0.19 (SD:0.4). The number of annual outbreaks decresed 37\%. No patients showed progression of disability due to EDSS. Side effects: 9 patients developed lymphopenia: grade $1(12.5 \%)$, grade $2(37.5 \%)$ and grade $3(6.25 \%)$ and 1 patient was suspended for continuing with lymphopenia grade 2 more than 18 months.

Conclusion: Cladribine was associated with a decrease in the number of annual outbreaks and with not progression of the disease during the study. The side effect was lymphopenia, similar to that published in the pivotal trials. The patients presented an excellent adherence.

Disclosure of Interest: None Declared.

\section{PP22}

Evaluation of the prescriptive appropriateness of human albumin in a hospital organization in Lombardy towards the objectives of the National Blood Program 2016-2020

\section{N. Macioce $^{1, *}$, F. Festinese ${ }^{2}$, L. Castellazzi ${ }^{2}$, G. Cambiè ${ }^{2}$ ${ }^{1}$ HOSPITAL PHARMACY SPECIALISATION, MILANO, ${ }^{2}$ ASST di Lodi, Lodi, Italy}

Background and Objective: Following the DLgs 21.12.2007 n.261, the National Blood Program 2016-2020 (PNS) has been issued, with the aim of developing both plasma collection and a rational and an appropriate use of plasma-derived medicinal products (MPDs). Among MPDs, human albumin has multiple functions exploited in clinical practice and there is a progressive increase in demand. These 
functions depends on its structural integrity, which, if pathologically altered, makes necessary its exogenous administration. The aims of this work are to evaluate the prescriptive appropriateness of $20 \%$ albumin solutions in a Lombardy Hospital in 2018, and to compare the amount of albumin dispensed with the national and regional demand.

Design: The requests for the year 2018, divided between the Intensive Care Unit (ICU) and the remaining Operating Units (OU), were evaluated in terms of completeness of prescription, i.e. the presence or absence of the value of albuminemia, proteinemia, weight; considering a cutoff value for albuminemia of $2,5 \mathrm{~g} / \mathrm{dL}$ and for proteinemia of $5 \mathrm{~g} / \mathrm{dL}$. Requests with values greater than those were considered inappropriate since oncotic activity is maintained with an albuminemia value of $2 \mathrm{~g} / \mathrm{dL}$. Appropriateness was in according to the current AISF-SIMTI 2016 and SIMTI 2009 Guidelines.

Results: Analyzing 154 requests of ICU, only 50,5\% of prescriptions was appropriate. In most cases, no data on albuminemia or levels greater than $2,5 \mathrm{~g} / \mathrm{dL}$ were indicated. For the remaining OUs, an even lower level of completeness was observed $(28,25 \%)$ with 79 requests out of 231 with an albuminemia $>2,5 \mathrm{~g} / \mathrm{dL}$. The main clinical indications found were prevention of post-paracentesis circulatory dysfunction (Grade of evidence 1A) (0,86\% OU); prevention of renal failure after spontaneous bacterial peritonitis (1A) (0,65\% ICU; $1,73 \%$ OU); hepatorenal syndrome (1A) (1,30\% ICU; $8,23 \%$ OU); ascites refractory to appropriate diuretic therapy (C2) (12,99\% ICU; $46,32 \%$ OU); therapeutic plasmapheresis (C2) (2,59\% ICU); after surgical conditions $(\mathrm{C} 2+)$ ( 9,74\% ICU; 3,46\% OU); septic shock (C1) $(33,12 \%$ ICU). Remaining indications had weak strength of recommendation and low or very low level of evidence.

Conclusion: Prior to the drafting of the PNS, the National Health Institute (Istituto Superiore di Sanità) calculated a total standardized demand of 597,5 g per 1000 inhabitants in Italy and of $600 \mathrm{~g} / 1000$ inhabitants in Lombardy. This study found a demand of 695,62 g/ 1000 inhabitants in the considered structure. The self-sufficiency of albumin is strongly influenced by clinical appropriateness. Most of albumin is used for indications with poor levels of evidence. The PNS goals is to reduce this figure to $400 \mathrm{~g} / 1000$ inhabitants and corrective actions will be proposed, e.g. a better completeness in requests, a formal distinction between requests made by ICU and by other units, and above all, a greater collaboration between physicians and pharmacists.

Disclosure of Interest: None Declared.

\section{PP23}

Comparisons of the rationality of selection of BZDS prescribed to community-residing seniors in Spain and the Czech Republic: results from the InoMed and the EUROAGEISM $\mathrm{H} 2020$ projects

A. Magátová, ${ }^{1 *}$, P. Modamio ${ }^{2}$, E. Mariňo ${ }^{2}$, J. Braun-Vives ${ }^{2}$, A. Lukačišinová $^{1}$, J. Reissigová ${ }^{1}$, D. Fialová ${ }^{1}$ on behalf of InoMed project (reg. No: CZ.02.1.01/0.0/0.0/18_069/0010046, 2019-2022), EUROAGEISM H2020-MCSF-ITN764632 project, PROGRESS Q42, Faculty of Pharmacy, Charles University (KSKF-2 Assoc. Prof. Fialová) and SVV 260417

${ }^{1}$ Department of Social and Clinical Pharmacy,, Faculty of Pharmacy in Hradec Králové, Charles University, Hradec Králové, Czech Republic, ${ }^{2}$ Clinical Pharmacy and Pharmaceutical Care Unit. Department of Pharmacy and Pharmaceutical Technology and Physical Chemistry, Faculty of Pharmacy and Food Sciences, University of Barcelona, Barcelona, Spain

Background and Objective: Benzodiazepines are a group of medications frequently prescribed for the treatment of anxiety, panic disorders, insomnia and co-treatment of depression. Due to age- related changes, risks of adverse drug events are substantially higher in older patients. Appropriate selection, dosing and length of therapy in geriatric patients should be regularly checked. The aim of our study was to identify and compare in pilot analyses the prevalence of use and selection of active substances by physicians when prescribing BZDs to community-residing older patients in Spain and the Czech Republic.

Setting and Method: Data of 260 Spanish and 450 Czech seniors $65+$ were prospectively assessed in the EuroAgeism H2020 project in 2019 with the use of study protocols. Spanish sample consisted of patients assessed in 3 community pharmacies in Barcelona and Czech seniors were assessed in community pharmacies in 3 regionally different cities. For pilot description of major sample characteristics and BZD drug use, descriptive statistics were applied using R-software (version 4.0.3). Differences between study samples were set up at the probability level $\mathrm{p}<0.05$.

Main outcome measures: Prevalence of use of benzodiazepines in Spain and the Czech Republic, comparison of prescription patterns (particularly geriatric BZD drug selection) using Beers 2019 criteria. Results: In both study samples, there was comparable proportion of women, $64.6 \%$ and $62.4 \%$ in Spain and the Czech Republic, respectively $(\mathrm{p}=0.563)$. Mean age of the studied populations was 76.6 and 71.7 years $(\mathrm{p}<0.001)$. Polypharmacotherapy was detected in $41.2 \%$ in Spain and $24.9 \%$ in the Czech Republic $(\mathrm{p}<0.001)$. Excessive polypharmacotherapy was found in $13.1 \%$ and in $3.8 \%$ $(\mathrm{p}<0.001)$. In Spanish sample, $36.2 \%$ of seniors used at least 1 BZDs which was significantly higher prevalence than in the Czech Republic $(4.9 \%, \mathrm{p}<0.001)$. Among top 3 most frequently prescribed BZDs were documented 1/ in Spain: lorazepam in $16.5 \%$ of patients, lormetazepam in $6.5 \%$, alprazolam in $4.6 \%$ of patients; $2 /$ in the Czech Republic: zolpidem in $2.4 \%$ of patients, alprazolam in $1.1 \%$ and bromazepam in $0.7 \%$. The statistically significant differences $(<0.05)$ were found particularly in prescription of lorazepam, lormetazepam and diazepam.

Conclusion: Our pilot findings confirmed significant differences in BZD drug use among samples of seniors in Spain and the Czech republic (both in the extend of use and selection of active substances from BZD drug class). As the intension of new geriatric guidelines is to reduce excessive and inappropriate use of BZDs in older patients (particularly appropriately select safer active substances, geriatric dose, indication and length of drug therapy), there is a need for detail implicit evaluation of appropriateness of BZD prescribing particularly in community-residing Spanish older adults.

Disclosure of Interest: None Declared.

\section{PP24}

Developement of an organizational model for the preparation of the MRNA BNT162B2 vaccine: a single center experience of an Italian Hospital Pharmacy

G. Fazzina $^{1}{ }^{1 *}$, M. Bellero ${ }^{1}$, S. Masucci ${ }^{1}$, R. Borello ${ }^{1}$ on behalf of Laboratory technicians, P. Aluffi ${ }^{1}$ on behalf of Laboratory technicians, K. Bushkolj ${ }^{1}$ on behalf of Laboratory technicians, $M$. Coglitore $^{1}$ on behalf of Laboratory technicians, G. Carchia ${ }^{1}$ on behalf of Laboratory technicians, G. Galota ${ }^{1}$ on behalf of Laboratory technicians, A. Luchin ${ }^{1}$ on behalf of Laboratory technicians, E. Miotto ${ }^{1}$ on behalf of Laboratory technicians, M. Michelotti $^{2}$ on behalf of Laboratory technicians, D. Pennetta ${ }^{2}$ on behalf of Laboratory technicians, T. Comandone ${ }^{1}$, M. Fiordelisi ${ }^{1}$, M. Marcato ${ }^{1}$, B. M. A. Parola ${ }^{1}$, S. Scalpello ${ }^{1}$, M. C. Azzolina ${ }^{3}$, C. Macchiolo $^{3}$, A. Gasco ${ }^{1}$ on behalf of Director of Hospital Pharmacy

${ }^{1}$ Hospital Pharmacy, ${ }^{2}$ Analysis laboratory, ${ }^{3}$ Healt Management Unit, A.O. Mauriziano, Turin, Italy 
Background and Objective: mRNA bnt162B2 COVID-19 vaccine should be prepared by a healthcare professional using aseptic technique to ensure the sterility of the prepared dispersion ${ }^{(1)}$. Hospital's Health Management Unit decided to built, as quick response to the vaccination campaign, an organizational model (OM) in order to guarantee the quality of the drug to be administered, the safety of the population and health professionals of the hospital.

Design: Development of an $\mathrm{OM}$ for the preparation according to Good Manufacturing Practice (GMP) which includes the provision of suitable rooms, staff training and the drafting of a procedure from reception to administration of the vaccine.

Results: All stages (setting up-dilution of the vial and preparation of the syringe)in this procedure were carried out under a hood to ensure the asepticity of the process as required in Summary of Product Characteristics(SmPC). A suitable environment has been set up equipped with a HEPA filter laminar flow hood, a key-locked refrigerator $\left(2-8{ }^{\circ} \mathrm{C}\right)$ for defrosting and storing the drug and a sterile welding machine to seal it in syringe packages. Two laboratory technicians band a pharamacist were identified and trained for the preparation of the vaccine. In order to guarantee the traceability of the process, it was prepared a setup protocol using the CytoSIFOII program which allows to draw up a personalized work plan for each person for the 1st and 2nd dose and to report following data on the label: personal data, dose, volume, set-up time, doctor and pharmacist signatures.

Conclusion: The above designed method allowed us to prepare vaccine doses in safety, immediately before the administration and without any drug waste. Daily schedules been respected, administering approximately 8500 doses to date and enhancing the role of each staff involved.

(1)Reference: Comirnaty ${ }^{\circledR}$ SmPC.

Disclosure of Interest: None Declared.

\section{PP25}

Medical treatment costs of hospitalized elderly patients with COVID-19-patient center care performance

P. Sazdanovic ${ }^{1,2}$, L. Tasic ${ }^{3,4}$, S. M. Jankovic ${ }^{2,5}$, D. Baskic ${ }^{6,7}$, I. Tadic $^{8}$, S. Sesto ${ }^{8}$, D. R. Milovanovic ${ }^{2,5, *}$

${ }^{1}$ Department of Anatomy, University of Kragujevac Faculty of Medical Sciences, ${ }^{2}$ University Clinical Centre Kragujevac,

${ }^{3}$ University of Kragujevac Faculty of Medical Sciences, Kragujevac, ${ }^{4}$ Creative Health, Belgrade, ${ }^{5}$ Pharmacology and Toxicology, ${ }^{6}$ Centre for Molecular Medicine and Stem Cell Research, University of Kragujevac Faculty of Medical Sciences, ${ }^{7}$ Public Health Institute, Kragujevac, ${ }^{8}$ University of Belgrade, Faculty of Pharmacy, Belgrade, Serbia

Background and Objective: Population of age 65 or more years, mostly with comorbidities, could be seriously affected by COVID-19 pandemic. The economy burden of health insurance funds due to pandemic should be subject of future research and analyze. In addition, the patient centered care model and performance need to been evaluated, from social, clinical and economy perspective. The aim of this research is to analyze a hospital medical treatment cost of COVID-19 elderly patients, from patients centered care approach.

Setting and Method: Observational case-series study included 40 males and 35 females aged 65 or more years which had COVID-19 and had been hospitalized at the University Clinical Centre Kragujevac (Serbia), during 2020. Clinical and cost data were collected retrospectively from hospital records and patient's files. Direct medical treatment costs (in Serbian dinars, 1 Euro $=117.5783$ RSD, on January 4th 2020) include ambulatory and ward services, drugs and medical devices which were accounted according to official rules and prices of the National Health Insurance Fund of Serbia. Descriptive statistics and hypothesis testing was used for data analysis.

Main outcome measures: Direct medical treatment costs.

Results: The mean age of patients was 73.4 years (standard deviation $\mathrm{SD}=6.4$, range $65-88$, median 72 ). The median score of Charlson Comorbidity Index was 4 (interquartile range IQ $=2$ ). The medians of medical treatment costs were following: for ambulatory services 284 RSD (IQ = 180), ward services 105,653 RSD (IQ = 117,429), drugs 57,731 RSD (IQ = 110,816), medical devices 14,586 RSD $(\mathrm{IQ}=42,713)$ and total hospital treatment costs 188,246 RSD (IQ = 268,588). Median total cost were three times higher in group of patients who died $(350,456 \mathrm{RSD}, \mathrm{IQ}=423,573)$ than in patients who survived $(113,844 \mathrm{RSD}, \mathrm{IQ}=143,678)$ and the difference was statistically significant $(\mathrm{Z}=-4.276, \mathrm{p}<0.001)$.

Conclusion: Direct medical costs of treatment for hospitalized elderly people with COVID-19 were substantial, particularly for patients who succumbed to the infection. According to patients' centered care model performance, the medical treatments include all available resources in every clinical specific and serious condition. Disclosure of Interest: None Declared.

\section{PP26}

Tensions on immunoglobulin supply and COVID 19. What impact on the hospital's outpatient pharmacy activity?

M. Moussouni ${ }^{1}$, D. Paya ${ }^{1}$, A.-C. Gérout ${ }^{1, *}$, M. Cosson ${ }^{1}$, B. Gourieux ${ }^{1}$, B. Michel ${ }^{1}$

${ }^{1}$ Pharmacy, University Hospital of Strasbourg, STRASBOURG, France

Background and Objective: In 2018, a worldwide shortage of Immunoglobulins (Ig) was noted. From that year, in order to prioritize the use of Ig, hospital pharmacists monitored compliance with the recommendations issued by the National authority (Ministry of Health). In 2020, the COVID-19 pandemic worsened the situation regarding the availability of Ig. A quota was then imposed. The hospital outpatient pharmacy department was strongly impacted regarding the dispensation of subcutaneous $\mathrm{Ig}$ (SC Ig).

The aim of the study was to review prescriptions in order to rationalize the dispensation of SC Ig in the hospital outpatient pharmacy sector, to achieve a saving of medication and ultimately to allow treatments of all patients.

Design: All prescriptions of SC Ig were reviewed from mid-July to the end of December 2020. Dosages, indications and residual IgG levels were collected and analyzed to determine whether the treatments were appropriate or whether dosage reductions were feasible. A pharmaceutical intervention (PI) was performed when both the residual IgG level was greater than $6 \mathrm{~g}$ per liter and the patient did not have recurrent bacterial infections.

Results: 79 patients were treated with SC Ig and 331 prescriptions were analyzed. A recent IgG level was present for 245 cases. The treatment was deemed appropriate for 49 patients, while for 30 patients the pharmacist considered that a reduction in dosage was possible. A total of 34 PIs were performed with an acceptance rate of $76,5 \%$. In 4 cases, 2 PIs were performed for the same patient, allowing successive reductions. The physician also self-decreased the SC Ig dosage for 11 patients. These interventions resulted in a monthly saving of $213 \pm 78 \mathrm{~g}$ of SC Ig allowing an average financial saving of $8,759 \pm 3254$ euros per month. A suspension of treatment during the summer period was observed for 8 patients and 3 new patients had a treatment initiation.

Conclusion: The revision of SC Ig prescriptions has proven to be effective in reducing doses and should be continued. It is indeed important that hospital pharmacists be actively involved in decision- 
making regarding such medications and assist clinicians in formulating and adjusting medication regimens in a timely manner.

Disclosure of Interest: None Declared.

\section{PP27}

Inflammatory bowel diseases: prescribing trends of adalimumab biosimilars in An italian hospital

S. Nobili ${ }^{1, *}$, L. Gambitta ${ }^{1}$, G. De Vivo ${ }^{1}$, E. Calzavara ${ }^{1}$, E. Ciceri ${ }^{1}$, S. Vimercati ${ }^{1}$

${ }^{1}$ HOSPITAL PHARMACY, ASST FATEBENEFRATELLI SACCO, MILAN, Italy

Background and Objective: The incidence of Inflammatory Bowel Diseases (IBD) is rising steadily, turning malabsorption into a public health priority. Anti-Tumor Necrosis Factor (Anti-TNF) monoclonal antibodies such as adalimumab (ADA) increase the treatment options of patients with moderate to severe IBD refractory to corticosteroid/ immunosuppressive therapy. Even though several biosimilars of ADA reached the European market and showed great efficacy, switching IBD patients from ADA originator (ADA-O) to ADA biosimilars (ADA-B) is still controversial.

To assess the increase of biosimilar prescriptions in an Italian Hospital.

Design: We conducted a retrospective observational study from January 2019 to December 2020, by considering all IBD patients who received therapy with ADA. For each patient, dispensed treatments and switches from ADA-O to ADA-B and vice-versa were analysed. Data were extracted from an administrative database and collected in an Excel datasheet.

Results: In 2019, 86 patients were treated: 70 (81.4\%) with ADA-O and $16(18.6 \%)$ with ADA-B. Out of 70 patients, $36(51.4 \%)$ switched to ADA-B throughout the year. In 2020, the number of patients increased to 111: 32 patients $(28.8 \%)$ received ADA-O and 79 (71.2\%) ADA-B. 69/111 (62.2\%) continued the treatment from the previous year (27 with ADA-O and 42 with ADA-B), 42/111 (37.8\%) patients were naïve (5 started with ADA-O and 37 with ADA-B). Throughout the year, 6 patients switched to ADA-B and 3 shifted back to ADA-O. ADA-B prescription was five times higher in 2020 than in $2019(+282.8 \%)$.

Conclusion: For the last two years, Hospital Pharmacists have sensitized clinicians by means of continuous information and counselling work. Indeed, the increased administrations of ADA-B showed in the results, confirms the clinicians' positive prescription attitude towards biosimilars. The implementation of biosimilars plays an important role in improving access to biological drugs for an increased number of patients and enhancing financial sustainability of National Health System.

Disclosure of Interest: None Declared.

\section{PP28}

Replacement therapy with alpha-1-antitrypsin: case reports

C. Palomo Palomo ${ }^{1, *}$, D. GUERRA ESTEVEZ ${ }^{1}$, M. M. ROMERO ALONSO $^{1}$, J. ESTAIRE GUTIERREZ ${ }^{1}$

${ }^{1}$ PHARMACY, INFANTA ELENA HOSPITAL, HUELVA, Spain

Background and Objective: The hospital's Pulmonology Unit has been conducting the early detection and treatment of patients with alpha-1-antitrypsin deficiency (DAAT) for two years, which allows a diagnosis to be made at an earlier age and to develop more effective primary prevention strategies.To analyse the criteria for use and the effectiveness of replacement therapy with alpha-1-antitrypsin (AAT) in patients with moderate to severe DAAT.

Setting and Method: Retrospective observational study in which patients with DAAT treated with AAT until October 2020.The criteria used to access the treatment were: DAAT with deficient ZZ phenotype or rare deficient variants, non-smokers in the last 6 months, and pulmonary emphysema demonstrated by a ratio of FEV1 (forced expiratory volume in the first second)/FVC (forced vital capacity) $<70 \%$ and FEV $1<80 \%$. Effectiveness was evaluated as the fall in FEV1 per year and the reduction in the frequency of hospital admissions related to chronic obstructive pulmonary disease (EPOC). Main outcome measures: Demographic, clinical, analytical and pharmacotherapeutic variables were collected.

Results: 5 patients ( 3 men) were included, with a median age of 63.6 years (59-69). All patients started with AAT in 2019, except for one patient in treatment since 2011. All patients met the utilisation criteria (only two patients with phenotype ZZ), except for one patient without baseline spirometric data and after the start of treatment. In all patients, replacement therapy slowed the fall in annual FEV1: $22 \%$ versus $28 \%, 30 \%$ versus $32 \%, 39 \%$ versus $56 \%$ and $44 \%$ versus $50 \%$. In patients with FEV1 22-44\%, treatment slowed the fall in FEV1 $15.6 \mathrm{ml} /$ year (8.3-26.6). Only one patient was admitted to the pulmonology service despite treatment with AAT.

Conclusion: The criteria of use allowed the selection of those patients who could obtain the greatest benefit. Treatment with AAT limits the functional deterioration of patients with AATD from moderate to severe, although in patients with phenotypes of high risk associated with lung / liver disease it did not produce a significant improvement in FEV1 (two patients). Treatment reduced hospital admissions although long-term data are necessary.

Disclosure of Interest: None Declared.

\section{PP29}

Pharmacists' experiences on the provision of clinical services during the COVID-19 pandemic: a pan European qualitative study

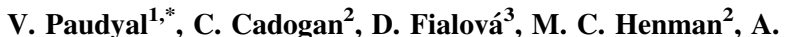

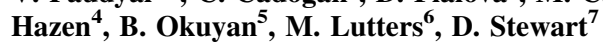

${ }^{1}$ University of Birmingham, Birmingham, United Kingdom, ${ }^{2}$ Trinity College Dublin, Dublin, Ireland, ${ }^{3}$ Charles University, Prague, Czech Republic, ${ }^{4}$ University of Manchester, Manchester, United Kingdom, ${ }^{5}$ Marmara University, Istanbul, Turkey, ${ }^{6}$ Swiss Federal Institute of Technology, Zurich, Switzerland, ${ }^{7}$ Qatar University, Doha, Qatar

Background and Objective: The pharmacy profession has an important role in the frontline healthcare response to COVID-19 across all settings. This study sought to explore the views and experiences of clinical pharmacists in relation to the provision of clinical pharmacy services during COVID-19.

Setting and Method: Semi-structured interviews were conducted with clinical pharmacists from across Europe. Invitations from professional organisations of clinical and hospitals pharmacists combined with a snowballing technique was used for participant recruitment. A topic guide was developed to conduct the interview using expertise of the research team and emerging literature. Thematic framework based on the The Pharmacy Emergency Preparedness and Response Framework and Disaster Preparedness Framework for pharmacy services was used to analyse the data.

Main outcome measures: Pharmacists' views, experiences and perceived impact of COVID-19 pandemic on clinical pharmacy services.

Results: A total of 22 participants from 16 European countries participated. They described a range of measures to protect patients, 
public and healthcare staff against COVID-19 transmission including developing and disseminating educational materials. They described facilitating clinical trials, gathering and appraising evidence and disseminating clinical information. Many hospital-based pharmacists were reassigned to help in intensive care. They described that routine clinical services were extensively interrupted and remote forms of communication were used. A number of facilitators and barriers related to uptake of new roles, recognition of pharmacists roles in the healthcare team, information gathering, communication with patients and healthcare professionals, and provision of routine clinical services were identified.

Conclusion: A range of service adaptations and adoption of novel roles to prevent and mitigate the public health impact of the pandemic were described by participants of this study. Results will be valuable in informing governments, public health agencies and healthcare systems in harnessing ongoing service provision and adapt to any future interruptions.

Disclosure of Interest: None Declared.

\section{PP30}

Potassium chloride potential errors with electronic prescriptions system: root cause analysis

\section{M. Romero Alonso ${ }^{1, *}$, D. Guerra Estevez ${ }^{1}$, C. Palomo Palomo $^{1}$, J. Estaire Gutiérrez ${ }^{1}$ \\ ${ }^{1}$ PHARMACY, INFANTA ELENA HOSPITAL, HUELVA, Spain}

Background and Objective: Electronic assisted prescription (hereinafter e-prescription) system improve patient safety and continuous control monitoring systems must always be implemented. When there are doubts in the e-prescription system, medication errors can occur. Potassium Chloride $(\mathrm{ClK})$ considered High Risk by the Institute for Safe Medication Practice, is the objective of this root cause analysis (RCA).

Ensure the correct use the correct use of drugs through the automated based-unit medication storage and distribution systems, regarding serum therapy with $\mathrm{ClK}$ addiction, both for physicians and nurses (permanente o eventual staff), after reporting 3 possible adverse events (AE)

Design: In June 2020, 3 potential AE (none of which have affected any patient) were communicated verbally to the pharmacist by a new physician, for raising questions/doubts about the information when the nurses read their electronic administration sheet (hereinafter e-worksheet), for serum therapy with $\mathrm{ClK}$. A 6-phase RAC is performed and an improvement plan is implemented to avoid confusion thereafter.

Results: Development of the RCA. Phase 1, problem identification: confusing reading of the e-worksheet nursing regarding the prescription of CLk. Phase 2, training of the work team: 2 pharmacists +1 physician +1 nurse. Phase 3 , information gathering: printing of the 3 e-worksheets nursing. Phase 4, map of the facts: doubtful information regarding the addiction of potassium chloride in relation to the frequency of administration. Phase 5, Ishikawa diagram with contributing factors: e-prescription system (frequency of the drug not in accordance with its association with serum therapy) + the internal organization (mostly permanent staff) + knowledge of the professionals (for permanent nursing staff, reading the e-worksheet nursing regarding $\mathrm{ClK}$ addiction does not generate any doubts, even if the frequency is not specified.).Phase 6 , development of solutions: changes in the settings of the e-electronic system, creation of 6 new frequencies for $\mathrm{ClK}$ in order to associate it according to the rhythm of the accompanying fluid therapy. Dissemination of a report on the changes/improvements made by email to physicians and nurses and informative talks, as reinforcement, in the nursing units.

Conclusion: Applications of multidisciplinary use, for example e-prescription system, must be continuously reviewed to ensure the prevention of AE. We have achieved the objective set by making changes to the application settings, thus avoiding risks in the administration of ClK.

Disclosure of Interest: None Declared.

\section{PP31}

Pharmacotherapeutic follow-up of omalizumab in chronic spontaneous urticaria

M. M. Romero Alonso ${ }^{1, *}$, C. Palomo Palomo ${ }^{1}$, D. Guerra Estevez $^{1}$, J. Estaire Gutierrez ${ }^{1}$

${ }^{1}$ PHARMACY, INFANTA ELENA HOSPITAL, HUELVA, Spain

Background and Objective: Omalizumab is the only authorized treatment for chronic spontaneous urticaria (CSU) refractory to conventional treatment (histamine $\mathrm{H} 1$ antagonist non-sedating-HH1ANSand corticosteroids). It's a drug of high economic impact so it's essential to monitor its use.

Analyze the use of omalizumab in CSU after approval according to previously established criteria and assessment of effectiveness based on a decrease in the use of HH1ANS and the Urticaria Activity Store (UAS) value at week 24 . The UAS value ranges from 0 (no pruritus) and 42 (severe pruritus).

Setting and Method: Descriptive analysis. 10 patients diagnosed with CSU were included. Data source: assisted electronic prescription, between 2017 and December 2020, for CSU and the digital medical record.

Main outcome measures: The variables collected are: sex, age, initiation of treatment with omalizumab, HH1ANS (drug name and dosage), corticosteroid treatment, UAS value after 2 doses, suspension (yes/no). The criteria for starting omalizumab are: no control of pruritus after histamine $\mathrm{H} 1$ antagonist doses up to 4 times the recommended dosage and UAS $>16$.

Results: Average age 39.7 years (20-65), 90\% female. Average time in treatment until December 2020: 23 months. In all patients, the antihistamine of choice was bilastine $40 \mathrm{mg} / 24 \mathrm{~h}$ pre-omalizumab and in $\mathrm{n}=6$ they also took a second antihistamine desloratadine, $80 \%$ of the total took corticosteroid therapy with deflazacort or prednisone. For 4 patients, UAS $=0$ was achieved after 2 doses of omalizumab ( $300 \mathrm{mg} / 4$ weeks) and at week 24 there was no need for the use of antihistamines, one of them was suspended omalizumab after an excellent response after almost 2 years in treatment. In another 4 patients the UAS has not reached 0 but they only take antihistamines on demand and very sporadically according to symptoms. One patient suspended treatment due to lack of response and another due to adverse reaction.

Conclusion: All patients have met the criteria to start omalizumab.According to the effectiveness data of our case series, the pharmacist could carry out an intervention to propose dose spacing in those patients who have been on treatment for more than 6 months and with UAS $=0$, since according to European public assessment report, the evidence is limited after that time, in order to improve efficiency.

Disclosure of Interest: None Declared. 


\section{PP32}

Preferred cobalamin supplementation to achieve sustained intake J.-P. Rothen ${ }^{1, *}$, D. Morina ${ }^{1}$, K. E. Hersberger ${ }^{1}$, I. Arnet ${ }^{1}$ on behalf of Pharmaceutical Care Research Group

${ }^{1}$ Pharmaceutical Sciences, University of Basel, Basel, Switzerland

Background and Objective: A cobalamin deficiency can have various causes such as dietary habits (vegetarian, vegan), advanced age, pernicious anaemia, gastrectomy, or drug interactions (metformin, proton pump blocker). Substitution is generally achieved through intramuscular administration, although oral supplementation is possible. In spite of limited active absorption, cobalamin is absorbed by passive diffusion

We investigated patients' and healthcare professionals' (HCPs) awareness of a potential cobalamin deficiency in the general population, currently used treatment, and preferred way of supplementation to achieve sustained intake and optimal adherence. Setting and Method: Focus group discussions (1) with patients having a potential cobalamin deficiency for various causes, and (2) with HCPs.

Main outcome measures: Patients' awareness of cobalamin deficiency, HCPs' diagnostic strategy for cobalamin deficiency, currently used cobalamin supplementation, and preferred way of supplementation to achieve optimal adherence. Statements were obtained with open-ended questions or presented with yes / no answer options.

Results: Nine individuals (mean age: 44 years; 3 men) and three HCPs (one general practitioner, one medical director of a nutrition center and one community pharmacist) attended a 1-h meeting each in March 2020. All patients were aware of a potential cobalamin deficiency, four $(44 \%)$ supplemented cobalamin in doses between 2.5 and $1000 \mu \mathrm{g}$. Three of them used supplements from abroad or the internet. Doctors usually prescribed intramuscular preparations in absence of alternative formulations. Oral administration was rated by six $(67 \%)$ of the patients as best for manifest and by eight (89\%) of them for subclinical deficiency. As oral formulation, eight (89\%) preferred tablets or capsules compared to drinking solution, four (44\%) preferred a weekly, five $(56 \%)$ a daily intake.

HCPs agreed that diagnosing cobalamin deficiency should equally be rooted in patient history, clinical symptoms and laboratory values. Intramuscular administration was rated by all three HCPs as best for manifest deficiency and by two of them in case of subclinical deficiency. They unanimously claimed for peroral reimbursable supplements so that individual therapies could be given to their patients.

Conclusion: Currently available cobalamin supplementation options in Switzerland only partially meet patients' and HCPs' needs.

Disclosure of Interest: None Declared.

\section{PP33}

Prevention of antiepileptic-related drug iatrogenicity in paediatrics: drafting of pamphlets for proper use

L. Scherer ${ }^{1, *}$, A. Fratta ${ }^{1}$, J. Lehrer ${ }^{1}$, P. Garzon ${ }^{2}$, M. H. Triboulet ${ }^{2}$, J. Agar $^{1}$

${ }^{1}$ Hospital Pharmacy, ${ }^{2}$ Neuropaediatry, Hôpital Armand Trousseau, Paris, France

Background and Objective: Within our establishment, the paediatric neurology department identified an antiepileptic drugs' misuse by patients and their parents. It can be explained by an overflow of misunderstood or unretained oral information. It affects all molecules, regardless of the galenic form.
The aim was to produce written documents for the families regarding proper use of antiepileptics in the paediatric population.

Design: A retrospective analysis of all prescribed antiepileptic molecules within the paediatric neurology department was conducted using a data retrieval of all prescriptions from July 1st to December 31 st, 2019 with Phedra ${ }^{\circledR}$ and Orbis ${ }^{\circledR}$ software programs.

Data from the Summary of Product Characteristics and leaflet were gathered. The most relevant pieces of information that should appear on the documents were validated by a working group of one pharmacy resident, two pharmacists, two paediatric neurologists and three paediatric nurses.

Results: Twenty antiepileptic molecules (ATC N03A) were found in 100 prescriptions (24\%). The four most prescribed antiepileptic drugs were chosen, in order to produce four pamphlets: sodium valproate $(40 \%)$, levetiracetam $(27 \%)$, carbamazepin $(23 \%)$ and vigabatrin (19\%).

Information was organized in eight categories: medical indication, modalities of use, different galenic forms, relevant interactions, frequent and unalarming side effects, adverse side effects, follow-up surveillance and tips for correct compliance. Pharmacists provided pharmaceutical knowledges, paediatric neurologists brought clinical aspects and nurses the practical knowledge of drug use. An additional paragraph allows the prescriber to add specific and individualized information.

Conclusion: Because of the COVID-19 containment mesures, teleconsultation have not permitted to provide those pamphlets to the families When possible, a satisfaction survey will be submitted to the families and the paediatric neurologists who were not in the working group.

With time, this action could be extended to others antiepileptic molecules and shared with hospital departments to limit misuse during hospitalization or to teach patients before discharge.

Disclosure of Interest: None Declared.

\section{PP34}

\section{Self-reported medication adherence in older patients}

S. Sesto ${ }^{1, *}$, I. Tadic ${ }^{1}$, V. Marinkovic ${ }^{1}$, M. Odalovic ${ }^{1}$, L. Tasic ${ }^{2,3}$, J. Brkic $^{4}$, D. Fialová ${ }^{4,5}$

${ }^{1}$ Department of Social Pharmacy and Pharmaceutical Legislation, University of Belgrade-Faculty of Pharmacy, Belgrade, ${ }^{2}$ University of Kragujevac-Faculty of Medical Sciences, Kragujevac, ${ }^{3}$ Creative Health, Belgrade, Serbia, ${ }^{4}$ Department of Social and Clinical Pharmacy, Faculty of Pharmacy in Hradec Kralove, Charles University, Hradec Kralove, ${ }^{5}$ Department of Geriatrics and Gerontology, 1st Faculty of Medicine, Prague, Czech Republic

Background and Objective: According to the Health Statistical Yearbook of the Republic of Serbia, during 2019, almost 20.6\% of the population was over 65 years. The number of comorbidities increases with advanced age, and consequently also numbers of medicines taken by geriatric patients. The aim of this study was to assess the subjectively reported medicines adherence in patients older than 65 . Setting and Method: This research analyzed Serbian data collected as part of the EuroAgeism ESR7 project using EuroAgeism ESR7 international study protocols for comprehensive geriatric assessment of older adults in pharmacy practices.

The Serbian sample included 450 patients over 65 years. Patients were recruited in community pharmacies in cities located in geographically different regions: Belgrade, Subotica and Nis (150 patients from each city), from May to December 2019. The selfreported medication adherence was estimated as a percentage value of the adherence to individual medications reported by the patient (from $0 \%$ as non/adherent to $100 \%$ as fully adherent). Ethical approvals 
were obtained from all community pharmacies and study was conducted according to all ethical principles.

Main outcome measures: Subjective Medication adherence in older patients and reasons for non/adherence.

Results: Most of patients were female $(59.1 \%)$ and was old in average 73.0 years (Range:65-93). The average number of prescribed medications was 5.0 (Range:1-14, Med = 5) and the most prevalent was cardiovascular disease $(89.1 \%)$. The average value of self-reported medication adherence was $92.4 \%(\mathrm{SD}=13.4)$. The most common reasons for non-adherence were forgetfulness (55.8\%), fear of side effects/addiction because of taking too many medications $(11.0 \%)$, high price $(8.7 \%)$, and complexity of medication regimen $(8.7 \%)$. Correlation between number of drugs and value of adherence was positive, but not statistically significant $(\rho=0.06, p>0.05)$.

Conclusion: This study has confirmed that older patients tend to take multiple medications in a day. Even though our expectation was that more drugs would affect medication adherence, the correlation was not statistically significant. Since forgetfulness is the most common cause of non-adherence, community pharmacists should remind patients more about the proper medication use.

Grant support of research group: European Union's Horizon 2020 research and innovation progamme under the Marie SkłodowskaCurie grant agreement No 764632. PROGRESS Q42, Faculty of pharmacy, Charles University.

Disclosure of Interest: None Declared.

\section{PP35}

Ustekinumab in Crohn's disease: experience in a regional hospital A. Trujillano,", M. A. Maestre ${ }^{1}$, N. Galán ${ }^{1}$, V. Cano ${ }^{1}$, A. Morego $^{1}$, M. Belló ${ }^{1}$, V. Llodrà ${ }^{1}$

${ }^{1}$ Pharmacy, Hospital de Manarcor, Manacor, Spain

Background and Objective: Ustekinumab(UST) has been approved for the treatment of Crohn's disease(CD) in patients who do not respond or are intolerant for conventional or other biological treatments.

To evaluate the effectiveness and safety of UST in patients with active CD.

Design: Retrospective observational study. Patients with active CD that received at least one induction dose of UST intravenously before September 2019 were included.
Demographic variables(sex and age), smoking habit, extraintestinal manifestations, location and behavior of the disease, perianal disease, previous abdominal surgery, previous biological treatments, maintenance schedule with UST after induction, concomitant immunosuppressants and/or corticosteroids were collected.

Effectiveness was analyzed by measuring C-reactive protein(CRP) and calprotectin at the beginning, at six months and one year after UST initial dose.

Adverse reactions collected in the medical history were reported to assess safety.

Results: $30 \mathrm{CD}$ patients were included. $67 \%$ were women with a mean age of $43.4 \pm 15.2$ years. $40 \%$ of the patients were smokers with extraintestinal illness in $30 \%$ of the cases. In terms of the location of the disease $50 \%$ was ileal, $33 \%$ ileocolonic, $13 \%$ colonic and $3 \%$ upper gastrointestinal tract, being $87 \%$ of inflammatory behavior.

$20 \%$ presented perianal disease, $27 \%$ previous abdominal surgery and $80 \%$ previously had received biological treatment (92\% 1-2 previous treatments and $8 \% 3$ prior).

$70 \%$ of patients continued with every 8 -weeks maintenance schedule with subcutaneous UST after induction, $10 \%$ with every 12 -weeks maintenance schedule and $20 \%$ continued with an intravenous dose of UST of $260 \mathrm{mg}$ (unauthorized indication according to the technical data sheet) $0.33 \%$ patients took concomitant immunosuppressants and $17 \%$ corticosteroids.

At 6 months of starting treatment $60 \%$ of patients decreased CRP and $69 \%$ calprotectin and a year after starting treatment $80 \%$ decreased CRP and 73\% calprotectin(calprotectin was not monitored in 4 patients).

$8 \%$ of patients reported adverse reactions( $31 \%$ arthralgias, $23 \%$ headaches, $15 \%$ infections, $15 \%$ pruritus, $8 \%$ herpes zoster and $8 \%$ blurred vision).

Conclusion: In our experience UST is an effective and safe alternative after treatment with other biological treatments and even in naïve patients with $\mathrm{CD}$.

In patients with reduced efficacy or intolerance to the subcutaneous route an intravenous maintenance dose can be an alternative.

It is important to protocol the analytical follow-up of patients to optimize the therapeutic and establish a personalized maintenance regimen.

Disclosure of Interest: None Declared.

Publisher's Note Springer Nature remains neutral with regard to jurisdictional claims in published maps and institutional affiliations. 